\title{
Five interpretations of Faà di Bruno's formula
}

\author{
Alessandra Frabetti (*), Dominique Manchon (**) \\ (*) Université de Lyon, Université Lyon 1, CNRS, \\ UMR 5208 Institut Camille Jordan, \\ Bâtiment du Doyen Jean Braconnier, \\ 43, blvd du 11 novembre 1918, F-69622 Villeurbanne Cedex, France. \\ email: frabetti@math.univ-lyon1.fr \\ (**) Université Blaise Pascal, CNRS, \\ UMR 6620, Laboratoire de Mathématiques, \\ BP 80026, \\ F-63171 Aubière Cedex, France. \\ email: manchon@math.univ-bpclermont.fr
}

Dedicated to Jean-Louis Loday

\begin{abstract}
In these lectures we present five interpretations of the Faà di Bruno formula which computes the $n$-th derivative of the composition of two functions of one variable: in terms of groups, Lie algebras and Hopf algebras, in combinatorics and within operads.
\end{abstract}

2010 Mathematics Subject Classification: 16T05, 05E15, 20G15, 22E65, 81R10

Key words: Proalgebraic groups, Hopf algebras, Operads

\section{Contents}

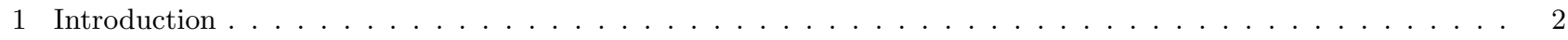

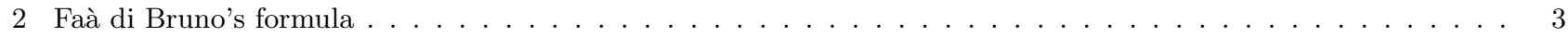

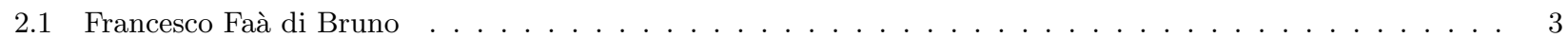

2.2 Faà di Bruno's and Lagrange's formulae . . . . . . . . . . . . . . . . . . . . . . . . . 3

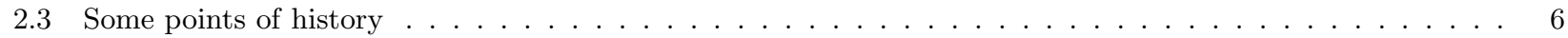

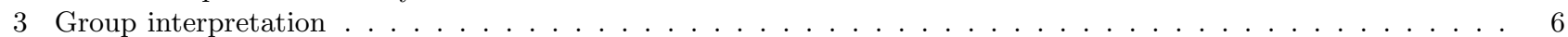

3.1 Formal diffeomorphisms $\ldots \ldots \ldots \ldots \ldots$

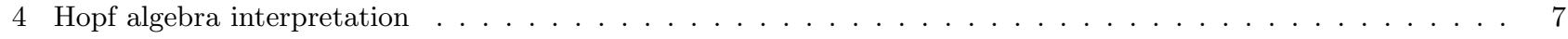

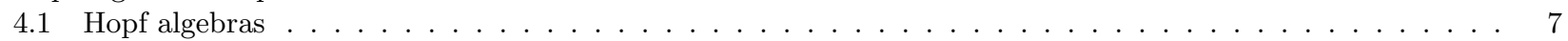

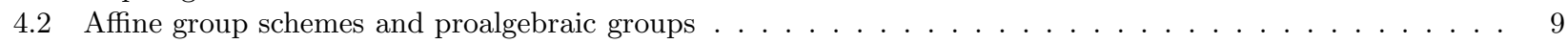

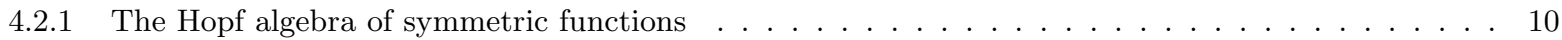


4.3 The Faà di Bruno Hopf algebra . . . . . . . . . . . . . . . . . . . . . . . . . . . . . . . . 11

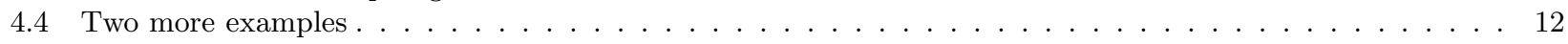

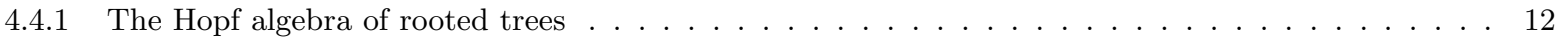

4.4 Feynman graphs and diffeographisms $[22] \ldots \ldots \ldots \ldots \ldots \ldots$

4.5 The non-commutative Faà di Bruno Hopf algebra . . . . . . . . . . . . . . . . . . . . . 15

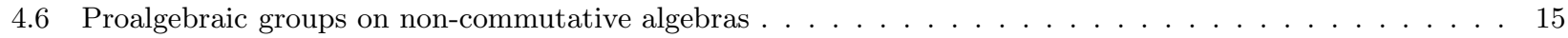

4.6 .1 Cogroups $[6] \ldots \ldots \ldots \ldots \ldots \ldots \ldots \ldots \ldots$

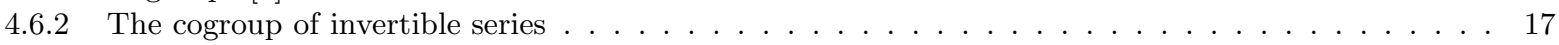

4.7 Open questions about the non-commutative Fà̀ di Bruno Hopf algebra . . . . . . . . . . . . . . 18

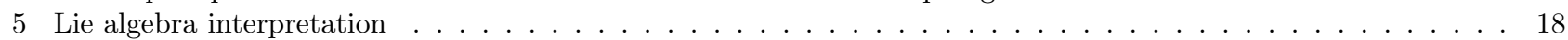

5.1 Vector fields, Witt and Virasoro Lie algebras . . . . . . . . . . . . . . . . . 18

5.2 Lie algebra of algebraic and proalgebraic groups . . . . . . . . . . . . . . . . . 19

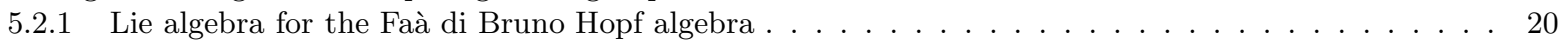

5.2 .2 Lie algebra for the Hopf algebra of rooted trees . . . . . . . . . . . . . . . . . . . 21

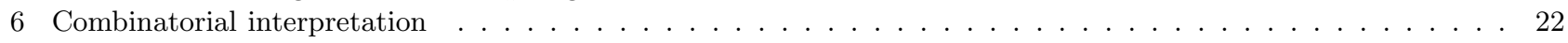

6.1 Incidence Hopf algebras . . . . . . . . . . . . . . . . . . . . . . . . . . . 22

6.1.1 The binomial and the divided power Hopf algebras . . . . . . . . . . . . . . . . . . 22

6.1 .2 The Faà di Bruno Hopf algebra . . . . . . . . . . . . . . . . . . . . . . . . . . 23

6.1 .3 The Hopf algebra of rooted trees . . . . . . . . . . . . . . . . . . . . . . . 23

6.2 Combinatorial Hopf algebras . . . . . . . . . . . . . . . . . . . . . . . . . . . 24

6.2.1 The non-commutative Faà di Bruno Hopf algebra and the brace bracket . . . . . . . . . . . . . . 25

6.2 .2 The Faà di Bruno Hopf algebra and the pre-Lie bracket . . . . . . . . . . . . . . . . . . 26

6.2 .3 The shuffle Hopf algebra . . . . . . . . . . . . . . . . . . . . . . . . 27

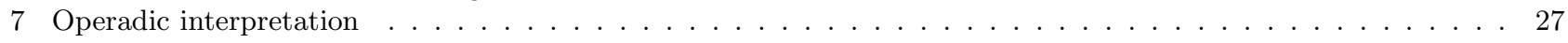

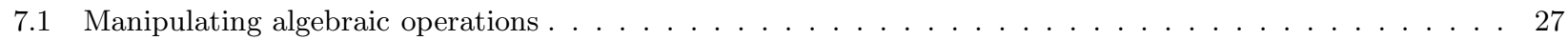

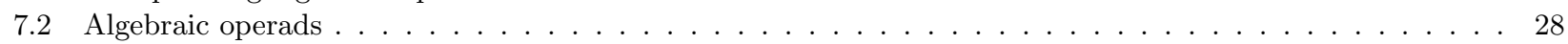

7.3 Pre-Lie algebras, Lie algebras and groups associated to operads . . . . . . . . . . . . . . . 30

7.3.1 The Faà di Bruno Hopf algebra and the associative operad . . . . . . . . . . . . . . . . . . 31

7.3.2 The QED charge Hopf algebra on planar binary trees and the duplicial operad . . . . . . . . . . 32

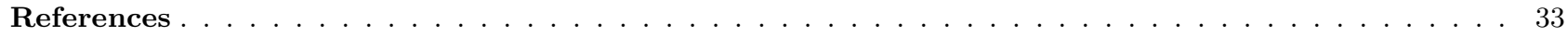

\section{Introduction}

In these lectures we present five interpretations of the Faà di Bruno formula which computes the $n$-th derivative of the composition of two functions of one variable.

(1) This formula tells explicitly how to compute the composition law in the group of formal diffeomorphisms in one variable. It is therefore related to the Lagrange inversion formula, which computes the inverse of a formal diffeomorphism in this group.

(2) In terms of Hopf algebras, it gives the coproduct of the so-called Faà di Bruno Hopf algebra, which can be seen as the coordinate ring of the previous group. It is one of the renormalization Hopf algebras which appeared recently in quantum field theory, and being related to the composition of formal series it is expected to appear, in its one-variable or several-variables form, in any perturbative theory. The Faà di Bruno Hopf algebra allows a non-commutative lift, but unlike what happens for the group of invertible formal series endowed with the product law, this non-commutative Hopf algebra does not represent a pro-algebraic group as a functor on non-commutative associative algebras. Its nature is then still an open question.

(3) The Lie algebra of the formal diffeomorphisms group is a well-known subalgebra of the Witt (and therefore also of the Virasoro) Lie algebra: the Faà di Bruno formula is therefore related to the well-known Lie bracket on these Lie algebras. The Faà di Bruno Hopf algebra is obtained from the Lie algebra of the formal diffeomorphisms group by considering the graded dual of its envoloping algebra (Cartier-Milnor-Moore correspondence). 
(4) In combinatorics, the Faà di Bruno Hopf algebra is an important example of incidence Hopf algebra [78]. It is also a right-sided combinatorial Hopf algebra [67], and therefore its associated Lie bracket is the commutator of a brace product. It would be interesting to extend this brace product to the Witt algebra, which is the Lie algebra of vector fields on the circle.

(5) Finally, an interpretation of the Faà di Bruno formula in operadic terms is also possible. We recall in Section ?? the definition of a prounipotent group (and its corresponding pronilpotent Lie algebra) associated to a large class of linear operad [18, 82], the group law being related to the operadic composition. Then, the group of formal diffeomorphisms (hence the Faà di Bruno formula) is the group associated to the operad Assoc governing associative algebras.

Acknowledgements We thank the referees for their careful reading and their pertinent suggestions which greatly helped us to improve the text.

\section{Faà di Bruno's formula}

\subsection{Francesco Faà di Bruno}

The "Cavaliere" Francesco Faà di Bruno was born on March 9, 1825, in Alessandria (Italy), and was the youngest of twelve children. He died on March 27, 1888, in Torino (Italy). He joined the Military Academy of Torino at the age of 15, took part in the first Italian independence war in 1848, and then went to Paris for two years, during which he studied mathematics with Augustin-Louis Cauchy and with the astronomer Urbain Jean Joseph Le Verrier. Back in Torino, he left the army in 1853. He gave mathematics lectures at Torino University and at the Military Academy, and was appointed Professor in 1876.

Besides his official teaching and research job, he carried out a lot of scientific and human activities such as mechanics (he invented, among other things, an electric alarm clock for his blind sister), music, and, above all, social and philanthropic activities inspired by his Christian faith. He became a catholic priest in 1876, and was beatified by John-Paul II in 1988 for his courageous fight against the hard living conditions of female workers in Torino in the 19th century. The interested reader can consult the biography by L. Giacardi [44] (in Italian).

\subsection{Faà di Bruno's and Lagrange's formulae}

Let us consider the set of smooth functions in one variable, endowed with the composition. If $f$ and $g$ are smooth functions of $t$, their composition is the smooth function $f \circ g$ defined by $(f \circ g)(t)=f(g(t))$. The Faà di Bruno formula tells how to compute the $n$-th derivative of the composite function $f \circ g$ in terms of the derivatives of $f$ and $g$. This famous formula was published by F. Faà di Bruno in the dense two-page paper "Sullo sviluppo delle funzioni" in 1855 [32], and says that

$$
\frac{\mathrm{d}^{n}}{\mathrm{~d} t^{n}} f(g(t))=\sum_{m=1}^{n} \sum \frac{n !}{k_{1} ! k_{2} ! \cdots k_{n} !} f^{(m)}(g(t))\left(\frac{g^{\prime}(t)}{1 !}\right)^{k_{1}}\left(\frac{g^{\prime \prime}(t)}{2 !}\right)^{k_{2}} \cdots\left(\frac{g^{(n)}(t)}{n !}\right)^{k_{n}}
$$

where the second sum is taken over the non-negative integers $k_{1}, \ldots, k_{n}$ such that $k_{1}+k_{2}+\cdots+k_{n}=m$ and $k_{1}+2 k_{2}+\cdots+n k_{n}=n$. For instance, for $n=3$, there are three possible sequences of such numbers: $\left(k_{1}, k_{2}, k_{3}\right)=(0,0,1),(1,1,0)$ and $(3,0,0)$. Then

$$
\begin{aligned}
\frac{\mathrm{d}^{3}}{\mathrm{~d} t^{3}} f(g(t)) & =\frac{3 !}{1 !} f^{\prime}(g(t)) \frac{g^{\prime \prime \prime}(t)}{3 !}+\frac{3 !}{1 !} f^{\prime \prime}(g(t)) \frac{g^{\prime}(t)}{1 !} \frac{g^{\prime \prime}(t)}{2 !}+\frac{3 !}{3 !} f^{\prime \prime \prime}(g(t)) \frac{g^{\prime}(t)^{3}}{(1 !)^{3}} \\
& =f^{\prime}(g(t)) g^{\prime \prime \prime}(t)+3 f^{\prime \prime}(g(t)) g^{\prime}(t) g^{\prime \prime}(t)+f^{\prime \prime \prime}(g(t)) g^{\prime}(t)^{3} .
\end{aligned}
$$

The most direct approach at this stage is to prove (2.1) directly by induction on $n$. Although it does not show particular difficulties, it is rather cumbersome. A very elegant alternative proof of (2.1) can be found in [38] (Proposition 8.3.4 therein), which heuristically goes as follows: we can extend any smooth function $f$ to a 
smooth functional $\widetilde{f}$ from $\mathbb{R}[[y]]$ to $\mathbb{R}[[y]]$ by Taylor expansion:

$$
\begin{aligned}
\tilde{f}\left(\sum_{k \geq 0} t_{k} y^{k}\right) & =\tilde{f}\left(t_{0}+\sum_{k \geq 1} t_{k} y^{k}\right) \\
& :=\sum_{n \geq 0} \frac{\left(\sum_{k \geq 1} t_{k} y^{k}\right)^{n}}{n !} \partial^{n} f\left(t_{0}\right) .
\end{aligned}
$$

Functionals like $T \mapsto \widetilde{f}(T)$ can be seen as smooth functions depending on the infinite set of variables $\left\{t_{0}, t_{1}, t_{2}, \ldots\right\}$, with $T=t_{0}+t_{1} y+t_{2} y^{2}+\cdots$. Set now $\partial_{k}:=\frac{d}{d t_{k}}$ for short and collect all the powers $\partial_{0}^{n}$ in the formal translation operator $e^{y \partial_{0}}=\sum_{k \geq 0} \frac{y^{k}}{k !} \partial_{0}^{k}$. The crucial properties:

$$
\begin{aligned}
\widetilde{f \circ g} & =\widetilde{f} \circ \widetilde{g} \\
e^{y \partial_{0}}(\tilde{f} \circ \widetilde{g})\left(t_{0}\right) & =\widetilde{f} \circ\left(e^{y \partial_{0}} \widetilde{g}\right)\left(t_{0}\right)=\widetilde{f \circ g}\left(t_{0}+y\right)
\end{aligned}
$$

together yield:

$$
\begin{aligned}
e^{y \partial_{0}}(\widetilde{f \circ g})\left(t_{0}\right) & =\widetilde{f}\left(\widetilde{g}\left(t_{0}+y\right)\right) \\
& =\widetilde{f}\left(g\left(t_{0}\right)+\sum_{k \geq 1} \frac{g^{(k)}\left(t_{0}\right)}{k !} y^{k}\right) \\
& =e^{\left(\sum_{k \geq 1} \frac{g^{(k)}\left(t_{0}\right)}{k !} y^{k}\right) \frac{d}{d t_{0}}} \tilde{f}\left(g\left(t_{0}\right)\right)
\end{aligned}
$$

Formula (2.1) is then obtained by taking $n$ ! times the coefficient of $y^{n}$ in (2.4) above. Formula (2.4) is valid in greater generality, replacing $\partial_{0}$ with any derivation $X$, with $g^{(k)}$ now standing for $X^{k}(g)$.

In order to make the proof above completely rigorous, we have to prove properties (2.2) and (2.3). A proof of these can be found in [38], based uniquely on formal-variable calculus (see [38, Sections 2.1, 2.2, 8.1-8.3]). One far-reaching application of this formal-variable calculus is the associativity principle for lattice vertex operators ([38], Equation (8.4.32), Theorem 8.4.2, Theorem 8.8.9 and Proposition 8.10.5). We won't go further into this direction, referring the interested reader to $[38,63]$ for an introduction to the theory of vertex operator algebras. See also [56] for a different approach to this vast topic.

Our interpretation of the formal variable argument alluded to above is the following: for any Taylor expansion functional $F=\widetilde{f}$ as above, where $f: \mathbb{R} \rightarrow \mathbb{R}$ is a smooth function, and for any $S, T \in \mathbb{R}[[y]]$ we have:

$$
F(T+y S)=e^{y S \partial_{0}} F(T) .
$$

The case $T=t_{0} \in \mathbb{R}$ is a simple rephrasing of the definition, and the case for general $T$ follows easily. Taking the linear part with respect to $S$ in $(2.5)$ successively for $S=1, y, y^{2}, \ldots$, we get an explicit expression for the differential $D F$ of $F$ at $T$ in terms of $D F(T)\left[\partial_{0}\right]$. Namely, for any $k \geq 1$ :

$$
D F(T)\left[\partial_{k}\right]=y^{k} D F(T)\left[\partial_{0}\right] .
$$

We can identify $\mathbb{R}[[y]]$ with its tangent space at each point $T$, via $\partial_{k} \simeq y^{k}$. We have then a coordinate-free formulation of $(2.6)$ :

$$
D F(T)[S]=S . D F(T)\left[\partial_{0}\right]
$$

for any $S \in \mathbb{R}[[y]]$. Conversely, any smooth functional $F: \mathbb{R}[[y]] \rightarrow \mathbb{R}[[y]]$ which satisfies (2.7) also satisfies the following identities involving higher-order differentials:

$$
D^{r} F(T)\left[S_{1}, \ldots, S_{r}\right]=S_{1} \cdots S_{r} \cdot D^{r} F(T)\left[\partial_{0}, \ldots, \partial_{0}\right] .
$$

Writing down the Taylor formula for the one-variable function $h \mapsto F(T+h y S)$ we see that (2.6) implies (2.5). Hence any $F$ verifying $(2.6)$ can be written as $F=\widetilde{f}$, with $\widetilde{f}\left(t_{0}\right)=F\left(t_{0}\right)$ for any $t_{0} \in \mathbb{R}$. Now consider two 
functionals $F=\widetilde{f}$ and $G=\widetilde{g}$. We claim that $F \circ G$ verifies (2.6): indeed,

$$
\begin{aligned}
D(F \circ G)(T)\left(\partial_{k}\right) & =D F(G(T)) \circ D G(T)\left[\partial_{k}\right] \\
& =D F(G(T)) \circ y^{k} D G(T)\left[\partial_{0}\right] \\
& =y^{k} D F(G(T)) \circ D G(T)\left[\partial_{0}\right] \\
& =y^{k} D(F \circ G)(T)\left[\partial_{0}\right] .
\end{aligned}
$$

Hence there exists $h \in C^{\infty}(\mathbb{R})$ such that $F \circ G=\widetilde{h}$, and evaluating it at $t_{0} \in \mathbb{R}$ yields $h=f \circ g$, which proves (2.2). Property (2.3) follows immediately:

$$
\begin{aligned}
\widetilde{f} \circ e^{y \partial_{0}} \widetilde{g}\left(t_{0}\right) & =\widetilde{f} \circ e^{\partial_{1}} \widetilde{g}\left(t_{0}\right) \\
& =\widetilde{f} \circ \widetilde{g}\left(t_{0}+y\right) \\
& =e^{\partial_{1}}(\widetilde{f} \circ \widetilde{g})\left(t_{0}\right) \\
& =e^{y \partial_{0}}(\widetilde{f} \circ \widetilde{g})\left(t_{0}\right) .
\end{aligned}
$$

Surprisingly enough, F. Faà di Bruno wrote this formula almost a century after a similar one was established: the equally famous Lagrange inversion formula of 1770 [62], which computes the compositional inverse of a smooth function. If $u=f(t)$ and $t=g(u)$ are two smooth functions, inverse to each other, with $f(0)=g(0)=0$ and $f^{\prime}(0) \neq 0$, then Lagrange's inversion formula says that, for $u$ in a neighborhood of zero,

$$
g(u)=\left.\sum_{n=1}^{N} \frac{1}{n !} \frac{\mathrm{d}^{n-1}}{\mathrm{~d} t^{n-1}}\left(\frac{f(t)}{t}\right)^{-n}\right|_{t=0} u^{n}+O\left(u^{N+1}\right)
$$

for any $N \geq 0$. For instance, for $u=f(t)=t e^{t}$, this formula allows us to compute $t$ as

$$
t=g(u)=\sum_{n=1}^{\infty}(-1)^{n-1} \frac{n^{n-1}}{n !} u^{n}
$$

because

$$
\left.\frac{\mathrm{d}^{n-1}}{\mathrm{~d} t^{n-1}}\left(\frac{f(t)}{t}\right)^{-n}\right|_{t=0}=\left.\frac{\mathrm{d}^{n-1}\left(e^{-n t}\right)}{\mathrm{d} t^{n-1}}\right|_{t=0}=(-n)^{n-1}
$$

The series (2.10) defines Lambert's W-function on its domain of convergence, with radius of convergence equal to $1 / e$ (see e.g. [81]). Similarly to the logarithm, this function admits a multivalued analytic extension to the whole complex plane with $1 / e$ removed.

In the two-page paper [32], Faà di Bruno also expresses $\frac{\mathrm{d}^{n}}{\mathrm{~d} t^{n}} f(g(t))$ as the determinant of the following $n \times n$ matrix:

$$
\left(\begin{array}{cccccccc}
f g^{\prime} & (n-1) f g^{\prime \prime} & \left(\begin{array}{c}
n-1 \\
2
\end{array}\right) f g^{\prime \prime \prime} & \left(\begin{array}{c}
n-1 \\
3
\end{array}\right) f g^{(4)} & \cdots & \left(\begin{array}{c}
n-1 \\
n-3
\end{array}\right) f g^{(n-2)} & (n-1) f g^{(n-1)} & f g^{(n)} \\
-1 & f g^{\prime} & (n-2) f g^{\prime \prime} & \left(\begin{array}{c}
n-2 \\
2
\end{array}\right) f g^{\prime \prime \prime} & \cdots & \left(\begin{array}{c}
n-2 \\
n-4
\end{array}\right) f g^{(n-3)} & (n-2) f g^{(n-2)} & f g^{(n-1)} \\
0 & -1 & f g^{\prime} & (n-3) f g^{\prime \prime} & \cdots & \left(\begin{array}{c}
n-3 \\
n-5
\end{array}\right) f g^{(n-4)} & (n-3) f g^{(n-3)} & f g^{(n-2)} \\
\vdots & \vdots & \vdots & \vdots & \cdots & \vdots & \vdots & \vdots \\
0 & 0 & 0 & 0 & \cdots & -1 & f g^{\prime} & f g^{\prime \prime} \\
0 & 0 & 0 & 0 & \cdots & 0 & -1 & f g^{\prime}
\end{array}\right)
$$

Unlike the Faà di Bruno formula (2.1), this beautiful matrix formula seemingly did not get any echo among mathematicians, nor any further development. 


\subsection{Some points of history}

It has been pointed out that Formula (2.1) was certainly known before Faà di Bruno's paper [32]: quoting W. P. Johnson [53], several formulas of the same type were established in the middle of the 19th century, by Hoppe (1845), Meyer (1847), J.F.C. Tiburce Abadie alias T.A. (1850) and Scott (1861). Moreover, as highlighted by A. D. D. Craik [24], formula (2.1) appears nearly as such, as early as 1800, in the remarkable book of Louis François Antoine Arbogast (1759-1803), professor of Mathematics in Strasbourg [1, "Remarques", pp. 43-44]. This work on differential calculus for composite functions inspired in turn several French and British mathematicians of the first half of the 19th century, e.g. [58, 60]. To sum up, although we won't change a well-established terminology, let us cite the following sentence from the conclusion of Craik's historical survey [24]:

"Faà di Bruno's formula" was first stated by Arbogast in 1800, and it might as appropriately be named after one of the ten or more authors who obtained versions of it before Faà di Bruno. Only the determinantal formulation of it ought to be called "Faà di Bruno's formula".

In the middle of the 20th century the Faà di Bruno formula is enriched with a combinatorial interpretation. In 1946, J. Riordan recognizes the Bell polynomials [3]:

$$
\mathrm{B}_{n, m}\left(x_{1}, x_{2}, \ldots, x_{l}\right)=\sum_{\substack{k_{1}+k_{2}+\cdots+k_{l}=m, k_{1}+k_{2}+\cdots+l k_{l}=n}} \frac{n !}{k_{1} ! \cdots k_{l} !}\left(\frac{x_{1}}{1 !}\right)^{k_{1}} \cdots\left(\frac{x_{l}}{l !}\right)^{k_{l}}, \quad l=n-m+1
$$

and rewrites Faà di Bruno's formula as [76]:

$$
\frac{\mathrm{d}^{n}}{\mathrm{~d} t^{n}} f(g(t))=\sum_{m=1}^{n} f^{(m)}(g(t)) \mathrm{B}_{n, m}\left(g^{\prime}(t), g^{\prime \prime}(t), \ldots, g^{(n-m+1)}(t)\right) .
$$

In 1965, R. Frucht and G-C. Rota [39] propose a purely combinatorial formulation of Faà di Bruno's formula:

$$
\frac{\mathrm{d}^{n}}{\mathrm{~d} t^{n}} f(g(t))=\sum_{m=1}^{n} \sum f^{(m)}(g(t)) g^{\prime}(t)^{k_{1}} g^{\prime \prime}(t)^{k_{2}} \cdots g^{(n)}(t)^{k_{n}},
$$

where the second sum is taken over the partitions of $\{1, \ldots, n\}$ with $m$ blocks having $k_{i}$ blocks with $i$ elements. For instance, for $n=3$, there are 5 partitions, $\{1,2,3\},\{1\}\{2,3\},\{2\}\{1,3\},\{3\}\{1,2\}$ and $\{1\}\{2\}\{3\}$ :

- $\{1,2,3\}$ has a single block with 3 elements, therefore to this partition corresponds the sequence $\left(k_{1}, k_{2}, k_{3}\right)=$ $(0,0,1)$;

- $\{1\}\{2,3\},\{2\}\{1,3\}$ and $\{3\}\{1,2\}$ have two blocks, one with 1 element and the other one with 2 , therefore there are three sequences $\left(k_{1}, k_{2}, k_{3}\right)=(1,1,0)$;

- $\{1\}\{2\}\{3\}$ has three blocks with 1 element each, therefore there is a corresponding sequence $\left(k_{1}, k_{2}, k_{3}\right)=$ $(3,0,0)$.

The number of resulting sequences matches with the coefficient in Faà di Bruno's formula. The proof of the Frucht-Rota formula starting from Riordan's formula resides in the following combinatorial interpretation of the Bell polynomials: the coefficient of $x_{1}^{j_{1}} \cdots x_{l}^{j_{l}}$ in $B_{n, m}\left(x_{1}, \ldots x_{l}\right)$ (with $l=n-m+1$ ) is equal to the number of partitions of a set of cardinality $n$ with $j_{r}$ blocks of size $r, r \in\{1, \ldots, l\}$ (and therefore $m$ blocks altogether).

\section{Group interpretation}

\subsection{Formal diffeomorphisms}

The composition of functions of one variable is an associative operation, for which the identity $\operatorname{id}(t)=t$ is the unit. Therefore the set of smooth functions which have a compositional inverse forms a group, called the group of smooth diffeomorphisms on the real line, and denoted by $\operatorname{Diff}(\mathbb{R})$. Groups of diffeomorphisms on a manifold are infinite dimensional groups, not locally compact, with many connected components classified by the mapping class group. Even for the simplest ones, like $\operatorname{Diff}(\mathbb{R})$ or $\operatorname{Diff}\left(\mathbb{S}^{1}\right)$, very little is known. 
Up to composition by a translation, a diffeomorphism on the real line fixes the origin. Up to a scalar factor, one can also assume that a diffeomorphism is tangent to the identity at the fixed point. In other words, the group of diffeomorphisms of the real line is the semi-direct product of the group of diffeomorphisms tangent to the identity by the group of affine transformations.

Thinking in terms of Taylor expansions, we will rather consider the group of formal diffeomorphisms tangent to the identity:

$$
G^{\mathrm{dif}}(\mathbb{K})=\left\{f(t)=\sum f_{n} t^{n} \in \mathbb{K}[[t]], f_{0}=0 \text { and } f_{1}=1\right\}
$$

whose definition makes sense for any field $\mathbb{K}$ of characteristic zero. Faà di Bruno's formula then expresses the $n$-th coefficient of the series $f \circ g$ in terms of the coefficients of $f$ and $g$ :

$$
(f \circ g)_{n}=\frac{1}{n !}(f \circ g)^{(n)}(0)=\sum_{m=1}^{n} \sum \frac{m !}{k_{1} ! k_{2} ! \cdots k_{n} !} \quad f_{m} g_{1}^{k_{1}} \cdots g_{n}^{k_{n}},
$$

where the second sum runs over $n$-uples $\left(k_{1}, \ldots, k_{n}\right)$ of non-negative integers such that $k_{1}+k_{2}+\cdots+k_{n}=m$ and $k_{1}+2 k_{2}+\cdots+n k_{n}=n$. In terms of Bell's polynomial, this coefficient becomes:

$$
(f \circ g)_{n}=\sum_{m=1}^{n} \frac{m !}{n !} \quad f_{m} B_{n, m}\left(g_{1}, 2 ! g_{2}, \ldots,(n-m+1) ! g_{n-m+1}\right) .
$$

Formula (3.2) can be directly checked by computing the coefficient of $t^{n}$ in the composition of the two formal series:

$$
(f \circ g)(t)=\sum_{m \geq 1} f_{m}\left(\sum_{k \geq 1} g_{k} t^{k}\right)^{m}
$$

expanding each power by means of the multinomial formula. The Faà di Bruno formula (2.1) is then directly derived from (3.2).

This group admits finite dimensional representations, none of which is faithful. Its smallest faithful representation is infinite dimensional, and presents $G^{\text {dif }}(\mathbb{K})$ as the group of infinite matrices of the form

$$
M(g)=\left(\begin{array}{cccccc}
g_{1} & g_{2} & g_{3} & g_{4} & g_{5} & \ldots \\
0 & g_{1} & 2 g_{2} & g_{2}^{2}+2 g_{3} & 2 g_{4}+2 g_{2} g_{3} & \ldots \\
0 & 0 & g_{1} & 3 g_{2} & 3 g_{3}+3 g_{2}^{2} & \ldots \\
0 & 0 & 0 & g_{1} & 4 g_{2} & \ldots \\
0 & 0 & 0 & 0 & g_{1} & \ldots \\
\vdots & & & \ldots & & \ddots
\end{array}\right),
$$

with $g_{1}=1$ here. In view of formula $(3.3)$, the coefficients $\left(M(g)_{i j}\right)_{i \leq j}$ are defined as:

$$
M(g)_{i j}=\frac{j !}{i !} B_{i, j}\left(g_{1}, 2 ! g_{2}, \ldots,(i-j+1) ! g_{i-j+1}\right) .
$$

\section{Hopf algebra interpretation}

\subsection{Hopf algebras}

We give here a brief account of the subject. A standard reference on Hopf algebras is Sweedler's monograph [80]. An associative unital algebra, which we will abbreviate as algebra (in the symmetric monoidal category of vector spaces over a field $\mathbb{K}$, the morphisms being linear maps), is a $\mathbb{K}$-vector space $A$ together with: 
- a multiplication $m: A \otimes A \longrightarrow A,(a, b) \mapsto m(a, b)=a \cdot b$ such that $m(m \otimes$ id $)=m($ id $\otimes m)$, i.e.

$$
(a \cdot b) \cdot c=a \cdot(b \cdot c) \quad \text { for any } a, b \in A
$$

- a unit $i: \mathbb{K} \hookrightarrow A$ such that $i(t s)=i(t) \cdot i(s)$ for any $s, t \in \mathbb{K}$ and such that, if we set $1_{A}=i\left(1_{\mathbb{K}}\right)$, we have $a \cdot 1_{A}=a=1_{A} \cdot a$ for any $a \in A$.

A (coassociative counital) coalgebra is a $\mathbb{K}$-vector space $C$ together with:

- a comultiplication (or coproduct) $\Delta: C \longrightarrow C \otimes C, c \mapsto \Delta(c)=\sum c_{(1)} \otimes c_{(2)}$ such that $(\Delta \otimes \mathrm{id}) \Delta=$ $($ id $\otimes \Delta) \Delta$.

- a counit $\varepsilon: C \longrightarrow \mathbb{K}$ such that $(\varepsilon \otimes I) \Delta=(I \otimes \varepsilon) \Delta=\operatorname{Id}_{C}$.

For example, if $C$ is any coalgebra with comultiplication $\Delta$ and counit $\varepsilon$, its linear dual $A=C^{*}$ is an algebra with product $m=\Delta^{*}$ and unit $i=\varepsilon^{*}$. Similarly, if $A$ is a finite-dimensional algebra with product $m$ and unit $i$, then $C=A^{*}=\operatorname{Hom}_{\mathbb{K}}(A, \mathbb{K})$ is a coalgebra with comultiplication $\Delta=m^{*}: A^{*} \longrightarrow A^{*} \otimes A^{*} \cong(A \otimes A)^{*}$ given by $\Delta(f)(a \otimes b)=f(a \cdot b)$, and counit $\varepsilon=i^{*}: A^{*} \longrightarrow \mathbb{K}$ given by $\varepsilon(f)=f\left(1_{A}\right)$.

A Hopf algebra is a $\mathbb{K}$-vector space $H$ which is both an algebra and a coalgebra, such that:

- The comultiplication $\Delta$ and the counit $\varepsilon$ are algebra morphisms, i.e:

$$
\begin{aligned}
& \Delta(a \cdot b)=\Delta(a) \cdot \Delta(b), \quad \Delta(1)=1 \otimes 1 \\
& \varepsilon(a \cdot b)=\varepsilon(a) \cdot \varepsilon(b), \quad \varepsilon(1)=1,
\end{aligned}
$$

where the product on $H \otimes H$ is given by $\left(a_{1} \otimes a_{2}\right) \cdot\left(b_{1} \otimes b_{2}\right)=\left(a_{1} \cdot b_{1}\right) \otimes\left(a_{2} \cdot b_{2}\right)$ for any $a_{1}, a_{2}, b_{1}, b_{2} \in H$.

- There is a linear map $S: H \longrightarrow H$, called the antipode, such that $m(S \otimes$ id $) \Delta=i \varepsilon=m($ id $\otimes S) \Delta$.

- The antipode is both an antimorphism of algebras and an antimorphism of coalgebras, i.e., for any $a, b \in H$, we have

$$
\begin{aligned}
& S(a \cdot b)=S(b) \cdot S(a), \quad S\left(1_{H}\right)=1_{H}, \\
& \Delta(S(a))=(S \otimes S) \Delta^{o p}(a), \quad \varepsilon(S(a))=\varepsilon(a),
\end{aligned}
$$

where $\Delta^{o p}=\tau \circ \Delta$, with $\tau(u \otimes v)=v \otimes u$.

The dual $A^{*}$ of any finite-dimensional Hopf algebra $(A, m, i, \Delta, \varepsilon, S)$ is also a Hopf algebra, with mutiplication $\Delta^{*}$, comultiplication $m^{*}$, unit $\varepsilon^{*}$, counit $i^{*}$ and antipode $S^{*}$. Let us give some classical examples of Hopf algebras:

(1) The universal enveloping algebra $U(\mathfrak{g})$ of a Lie algebra $\mathfrak{g}$. By definition, $U(\mathfrak{g})=T(\mathfrak{g}) / J$ is the quotient of the free associative algebra on the vector space $\mathfrak{g}$ by the two-sided ideal $J$ generated by $\{x \otimes y-$ $y \otimes x-[x, y], x, y \in \mathfrak{g}\}$. The comultiplication, the counit and the antipode are the algebra morphisms (antimorphism for the antipode) given on the generators $x \in \mathfrak{g}$ by

$$
\Delta(x)=x \otimes 1+1 \otimes x, \quad \varepsilon(x)=0, \quad S(x)=-x .
$$

The elements of $\mathfrak{g}$ are said to be primitive in the Hopf algebra, because of the special form the comultiplication $\Delta$ takes on them. This Hopf algebra is cocommutative, i.e. $\Delta(u)=\Delta^{o p}(u)$ for any $u \in U(\mathfrak{g})$.

(2) The group algebra $\mathbb{K} G$ of a finite group $G$. By definition, $\mathbb{K} G$ is the vector space freely generated by the elements of $G$, with multiplication bilinearly induced from the group law. The comultiplication, the counit and the antipode are the algebra morphisms (antimorphism for the antipode) given on the generators $x \in G$ by

$$
\Delta(x)=x \otimes x, \quad \varepsilon(x)=\delta_{x, 1_{G}}, \quad S(x)=x^{-1} .
$$

The elements of $G$ are said to be group-like in the Hopf algebra, because of the special form the $\Delta$ takes on them. This Hopf algebra is also cocommutative. 
(3) The algebra of representative functions $R[G]$ of a group $G$. By definition, $R[G]$ is the algebra generated by the matrix elements of finite-dimensional representations of $G$. The remarkable property $R[G] \otimes R[G] \cong$ $\mathbb{R}[G \times G]$ (see [17, Lemma 3.1.1]) allows us to define the coproduct of a function (of one variable) as being a function of two variables. Explicitly, the coproduct, the counit and the antipode of any representative function $f$ on $G$ are defined as follows, for any $x, y \in G$ :

$$
\Delta(f)(x, y)=f(x y), \quad \varepsilon(f)=f\left(1_{G}\right), \quad S f(x)=f\left(x^{-1}\right) .
$$

This Hopf algebra is commutative and is also called the coordinate Hopf algebra of the group $G$.

If $G$ is a finite group, any function on $G$ is representative, and the Hopf algebra $R[G]$ is dual to the Hopf algebra $\mathbb{K} G$. If $G$ is a compact Lie group over $k=\mathbb{R}$, "almost all" functions on $G$ are representative, in the sense that there is a nondegenerate pairing $\langle\cdot, \cdot\rangle$ between $R[G]$ and the enveloping algebra $U(\mathfrak{g})$, where $\mathfrak{g}$ is the Lie algebra of $G$, such that $\langle\delta(f), u \otimes v\rangle=\langle f, u v\rangle$ and $\langle f \otimes g, \Delta u\rangle=\langle f g, u\rangle$ for any $f, g \in R[G]$ and any $u, v \in U(\mathfrak{g})$. The pairing is given by $\langle f, u\rangle:=u . f(e)$, where $u$ acts on $f$ as a (left-invariant) differential operator, and where $e$ is the unit of the group [17, 3.3 and 3.4].

\subsection{Affine group schemes and proalgebraic groups}

Let $H$ be a commutative Hopf algebra and $A$ a commutative unital algebra over some field $\mathbb{K}$. Then, the vector space $\mathcal{L}(H, A)$ of $\mathbb{K}$-linear maps from $H$ to $A$ inherits a canonical unital algebra structure. The multiplication is given by the convolution product, defined, for any two $\alpha, \beta \in \mathcal{L}(H, A)$ as

$$
\alpha * \beta:=m_{A} \circ(\alpha \otimes \beta) \circ \Delta_{H} .
$$

The convolution unit is the linear map $e_{A}:=i_{A} \circ \varepsilon_{H}$. The vector space $\mathcal{L}(H, A)$ is often huge and not easy to handle. However it contains a subgroup which is, roughly speaking, equivalent to $H$. In fact, let CAlg be the category of commutative and unital associative $\mathbb{K}$-algebras and consider the subset

$$
G_{H}(A):=\operatorname{Hom}_{\mathrm{CAlg}}(H, A)
$$

of $A$-valued characters of $H$, i.e. unital algebra morphisms from $H$ to $A$. It can easily be checked that the convolution of two characters is still a character and that the convolution inverse of a character is still a character, because $H$ and $A$ are commutative algebras. The unit $e_{A}=i_{A} \circ \varepsilon_{H}$ of $\mathcal{L}(H, A)$ is also a character, and the inverse is given by $\varphi \mapsto \varphi \circ S$ where $S$ is the antipode. This construction is functorial, that is, for any unital commutative algebras $A, B$ and for any commutative unital algebra morphism $f: A \rightarrow B$, there is a group morphism $G_{H}(f): G_{H}(A) \rightarrow G_{H}(B)$.

An affine group scheme [86] is a functor from the category of commutative algebras to the category of groups, representable by a commutative Hopf algebra $H$.

The group $G_{H}(A)$ has the particular property that $H$ can then be regarded as an algebra of $A$-valued functions on the group, because any element $h \in H$ can be seen as a function acting on $\alpha \in G_{H}(A)$ as $h(\alpha):=\alpha(h)$, and the fact that $\alpha$ is an algebra morphism, that is $\alpha\left(h \cdot h^{\prime}\right)=\alpha(h) \cdot \alpha\left(h^{\prime}\right)$ for any $h, h^{\prime} \in H$, guarantees that we recover the usual definition of the product of functions, that is, $\left(h \cdot h^{\prime}\right)(\alpha)=h(\alpha) \cdot h^{\prime}(\alpha)$. Moreover, the coproduct on $H$ then coincides with the coproduct on functions induced by the group law in $G_{H}(A)$, that is, $\Delta(h)(\alpha, \beta)=h(\alpha * \beta)$, because of the definition of the convolution product in $G_{H}(A)$. Therefore, any group $G_{H}(A)$ behaves like a compact Lie group, and $H$ like the algebra of its representative functions.

An algebraic group is by definition a functor $G$ from the category of commutative algebras to the category of groups, having the property that there exists a commutative and finitely generated Hopf algebra $H$ such that the group $G(A)$ is isomorphic to $G_{H}(A)$ for any commutative unital algebra $A$. In other words, an algebraic group is a representable functor from the category of commutative algebras to the category of groups, such that the Hopf algebra which represents it is finitely generated. In this case, the algebra $H$ is the algebra of $A$-valued polynomial functions on the group $G_{H}(A)$, and its generators can be seen as coordinate functions on the group. Classical examples of algebraic groups are the matrix groups $G L_{n}, S L_{n}, S O_{n}$ and $S U_{n}$. 
A pro-algebraic group is a projective limit of algebraic groups. It can be viewed as a representable functor from the category of commutative algebras to the category of groups, such that the Hopf algebra which represents it is an inductive limit of finitely generated Hopf algebras. Hence the group has infinitely many coordinate functions. As any commutative Hopf algebra is an inductive limit of finitely generated hopf algebras, any affine group scheme is pro-algebraic [86, Theorem 3.3].

Finally, a pro-algebraic group $G_{H}$ is called prounipotent (see e.g. [17, Paragraph 3.9]) if the Hopf algebra $H$ is connected, which means that there exists an increasing filtration of vector spaces

$$
H^{0} \subset \cdots \subset H^{n} \subset \cdots
$$

with $H=\bigcup_{n \geq 0} H^{n}$, such that the vector space $H^{0}$ is one-dimensional, i.e. $H^{0}=\mathbb{K} \cdot 1_{H}$, and such that the multiplication and the coproduct are of filtration degree zero, i.e. for any $p, q \geq 0$ we have

$$
H^{p} \cdot H^{q} \subset H^{p+q} \quad \text { and } \quad \Delta\left(H^{n}\right) \subset \sum_{p+q=n} H^{p} \otimes H^{q} .
$$

It is well known that the antipode of a connected Hopf algebra "comes for free" by recursive relations (in other words, any connected filtered bialgebra is a Hopf algebra), and is also of filtration degree zero, i.e. $S\left(H^{n}\right) \subset H^{n}$ for any $n \geq 0[33,71]$.

4.2.1 The Hopf algebra of symmetric functions A basic example of a pro-algebraic group is the group $G^{\text {inv }}$ of invertible series in one variable with pointwise multiplication: for any commutative unital algebra $A$, the group $G^{\text {inv }}(A)$ is the set of formal series

$$
f(t)=1_{A}+\sum_{n \geq 1} f_{n} t^{n}, \quad f_{n} \in A
$$

endowed with the product

$$
(f g)(t)=f(t) g(t)=1_{A}+\sum_{n \geq 1}\left(\sum_{k=0}^{n} f_{k} g_{n-k}\right) t^{n},
$$

with the convention $f_{0}=g_{0}=1_{A}$. Its representing Hopf algebra is the polynomial ring $\mathcal{H}_{\text {inv }}=\mathbb{K}\left[x_{1}, x_{2}, x_{3} \ldots\right]$ on infinitely many variables $x_{n}, n \geq 1$, which play the role of $A$-valued coordinate functions on each group $G^{\text {inv }}(A)$, that is,

$$
x_{n}: G^{\mathrm{inv}}(A) \rightarrow A, f \mapsto x_{n}(f):=f_{n} .
$$

The coproduct and the counit on $\mathcal{H}_{\text {inv }}$ are defined, for any $f, g \in G^{\text {inv }}(A)$, as

$$
\Delta_{\text {inv }}\left(x_{n}\right)(f, g)=x_{n}(f g) \quad \text { and } \quad \varepsilon\left(x_{n}\right)=x_{n}(1),
$$

where the constant series $1(t)=1_{A}$ is the unit in $G^{\mathrm{inv}}(A)$, and can be easily computed as

$$
\Delta_{\text {inv }}\left(x_{n}\right)=\sum_{p=0}^{n} x_{p} \otimes x_{n-p} \quad \text { and } \quad \varepsilon\left(x_{n}\right)=\delta_{n, 0},
$$

where we assume that $x_{0}=1$. The resulting bialgebra is graded, if we set the degree of a monomial $x_{n_{1}}^{d_{1}} \cdots x_{n_{k}}^{d_{k}} \in$ $\mathcal{H}_{\text {inv }}$ to be $\sum_{i=1}^{k} n_{i} d_{i}$, that is, if we require the generators $x_{n}$ to be graded, with degree $\left|x_{n}\right|=n$. In degree zero there are only scalars, therefore $\mathcal{H}_{\mathrm{inv}}$ is connected and the antipode is automatically defined by the recursive formula

$$
S\left(x_{n}\right)=-\sum_{p=0}^{n-1} S\left(x_{p}\right) x_{n-p} .
$$

Hence $G^{\text {inv }}$ is a pronilpotent group. The Hopf algebra $\mathcal{H}_{\text {inv }}$ is both commutative and cocommutative, and coincides with the well-known Hopf algebra of symmetric functions. 


\subsection{The Faà di Bruno Hopf algebra}

Another key example of a pro-algebraic group is the group $G^{\text {dif }}$ of formal diffeomorphisms tangent to the identity, and it is also prounipotent. Indeed, it is a functor: for any commutative unital algebra $A$, the group $G^{\text {dif }}(A)$ is the set of formal series with coefficients in $A$, of the form

$$
f(t)=t+\sum_{n \geq 2} f_{n} t^{n} \in t A[[t]]
$$

equipped with the usual composition law. It is the projective limit of the algebraic groups $G^{\text {dif }}(n)(A)$, for $n \geq 1$, which are the image of $G^{\mathrm{dif}}(A)$ in the quotient $t A[[t]] / t^{n+1} A[[t]]$. Furthermore, the functor $G^{\text {dif }}$ is representable: its representing Hopf algebra $H$ is the Hopf algebra of $A$-valued polynomial functions on $G^{\text {dif }}(A)$, freely generated as a commutatve algebra by the functions

$$
x_{n}: G^{\operatorname{dif}}(A) \rightarrow A, f \mapsto x_{n}(f):=f_{n+1}, \quad \text { for } n \geq 1 .
$$

The coproduct is induced by the composition of series:

$$
\Delta\left(x_{n}\right)(f \otimes g)=x_{n}(f \circ g) \text { for any } f, g \in G^{\mathrm{dif}}(A),
$$

counit is induced by the unit $\operatorname{id}(t)=t$ of the group:

$$
\varepsilon\left(x_{n}\right)=x_{n}(\mathrm{id}),
$$

and antipode is induced by the composition-inversion of series:

$$
S\left(x_{n}\right)=x_{n}\left(f^{-1}\right) \quad \text { for any } f \in G^{\operatorname{dif}}(A) .
$$

The Lagrange inversion formula (2.9) gives an explicit formula for $S\left(x_{n}\right)$. Using eq. (3.2) we can explicitly compute the coproduct. Up to a flip between left and right terms in the tensor product $H \otimes H$ (caused by the fact tht we write composition of functions from right to left), this Hopf algebra coincides with the Faà di Bruno Hopf algebra

$$
\mathcal{H}_{\mathrm{FdB}}=\mathbb{K}\left[x_{1}, x_{2}, x_{3}, \ldots\right]
$$

with coproduct and counit given, on the generators, by

$$
\begin{aligned}
& \Delta_{\mathrm{FdB}}\left(x_{n}\right)=\sum_{m=0}^{n} \frac{(m+1) !}{(n+1) !} \mathrm{B}_{n+1, m+1}\left(1,2 ! x_{1}, 3 ! x_{2}, \ldots,(n-m+1) ! x_{n-m}\right) \otimes x_{m} \\
& =\sum_{m=0}^{n}\left(\sum_{\substack{k_{0}+k_{1}+\cdots+k_{n}=m+1, k_{1}+2 k_{2}+\cdots+n k_{n}=n-m}} \frac{(m+1) !}{k_{0} ! k_{1} ! \cdots k_{n} !} x_{1}^{k_{1}} \cdots x_{n}^{k_{n}}\right) \otimes x_{m}, \\
& \varepsilon\left(x_{n}\right)=\delta_{n, 0},
\end{aligned}
$$

where we use the convention $x_{0}=1$, and where B denotes the Bell polynomial. The antipode can be found recursively, because $\mathcal{H}_{\mathrm{FdB}}$ is a graded and connected bialgebra, where the generators have degree $\left|x_{n}\right|=n$, for $n \geq 0$. The left-right flip of factors means that $\mathcal{H}_{\mathrm{FdB}}$ is in fact the representative Hopf algebra of the opposite pro-algebraic group $\left(G^{\text {dif }}\right)^{o p}$, with opposite composition law $f \circ^{o p} g=g \circ f$. For any commutative algebra $A$, the group $G^{\mathrm{dif}}(A)$ can then be seen as the group of $A$-valued characters of $\mathcal{H}_{\mathrm{FdB}}$ with reversed convolution product: any formal series $f \in G^{\mathrm{dif}}(A)$ is identified with a linear map $f: \mathcal{H}_{\mathrm{FdB}} \rightarrow A$ satisfying the properties

$$
f(1)=1_{A} \quad \text { and } \quad f(x y)=f(x) f(y) \quad \text { for any } x, y \in \mathcal{H}_{\mathrm{FdB}}
$$

The composition of two series $f, g \in G^{\operatorname{dif}}(A)$, then, coincides with their opposite convolution product, namely

$$
f \circ g=m_{A} \circ(g \otimes f) \circ \Delta_{\mathrm{FdB}}
$$

Detailed explanations can also be found in [34, Section II]. The Faà di Bruno Hopf algebra first appeared under this name in 1974 (P. Doubilet, [25]), and in 1979 (S.A. Joni and G.-C. Rota, [54]), as an example of a Hopf algebra structure obtained from the lattice of set partitions. We say more about such Hopf algebras in section 6. It is also worth mentioning that the Faà di Bruno Hopf algebra is a Hopf subalgebra of the 
Connes-Moscovici Hopf algebra, which is neither commutative nor cocommutative (see [23, Paragraph III] for a detailed account, see also [74]). The Faà di Bruno Hopf algebra also plays a role in applied mathematics, e.g. in control theory [48].

\subsection{Two more examples}

4.4.1 The Hopf algebra of rooted trees [20, 35, 71] A rooted tree is a class of oriented (non planar) graphs with a finite number of vertices, among which one is distinguished and called the root, such that any vertex admits exactly one incoming edge, except the root which has no incoming edges. Any tree yields a poset structure on the set of its vertices: two vertices $x$ and $y$ verify $x \leq y$ if and only if there is a path from a root to $y$ passing through $x$. Two graphs are equivalent (hence define the same rooted tree) if and only if the two underlying posets are isomorphic. Here is the list of rooted trees up to five vertices:

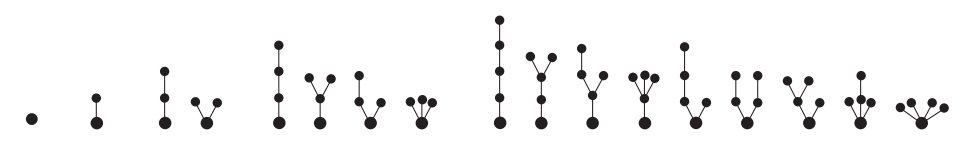

A rooted forest is a finite collection of rooted trees. The empty set is the forest with containing no trees, and is denoted by $\mathbf{1}$. The grafting operator $B_{+}$takes any forest and returns the tree obtained by grafting all components onto a common root. In particular, $B_{+}(\mathbf{1})=\bullet$.

Let $\mathcal{T}$ denote the set nonempty rooted trees and let $\mathcal{H}_{\mathrm{RT}}=\mathbb{K}[\mathcal{T}]$ be the free commutative and unital algebra generated by the elements of $\mathcal{T}$. We identify a product of trees with the forest consisting of these trees. Therefore the vector space underlying $\mathcal{H}_{\mathrm{RT}}$ is the linear span of rooted forests. This algebra is a graded and connected Hopf algebra, called the Hopf algebra of rooted trees, with the following structure. The grading is given by the number of vertices of trees. The coproduct on a rooted forest $u$ (i.e. a product of rooted trees) is described as follows: the set $\mathcal{V}(u)$ of vertices of a forest $u$ is endowed with the partial order defined by $x \leq y$ if and only if there is a path from a root to $y$ passing through $x$. Any subset $W$ of the set of vertices $\mathcal{V}(u)$ of $u$ defines a subforest $u_{\left.\right|_{W}}$ of $u$ in an obvious manner, i.e. by keeping the edges of $u$ which link two elements of $W$. The poset structure is given by restriction of the partial order to $W$, and the minimal elements are the roots of the subforest. The coproduct is then defined by:

$$
\Delta_{\mathrm{RT}}(u)=\sum_{\substack{V \amalg W=\mathcal{V}(u) \\ W<V}} u_{\left.\right|_{V}} \otimes u_{\left.\right|_{W}}
$$

Here the notation $W<V$ means that $y<x$ for any vertex $x$ in $V$ and any vertex $y$ in $W$ such that $x$ and $y$ are comparable. Note that both $\emptyset<V$ and $V<\emptyset$. Such a couple $(V, W)$ is also called an admissible cut, with crown (or pruning) $u_{\left.\right|_{V}}$ and trunk $u_{\left.\right|_{W}}$. We have for example:

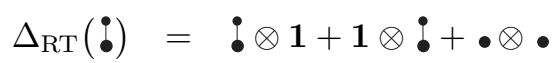

$$
\begin{aligned}
& \Delta_{\mathrm{RT}}(\dot{\bullet})=\ddot{\bullet} \otimes \mathbf{1}+\mathbf{1} \otimes \ddot{\bullet}+2 \bullet \otimes \mathfrak{\bullet}+\bullet \otimes \bullet .
\end{aligned}
$$

The counit is $\varepsilon(\mathbf{1})=1$ and $\varepsilon(u)=0$ for any non-empty forest $u$. The coassociativity of the coproduct is easily checked using an iterated formula for the restricted coproduct

$$
\widetilde{\Delta}_{\mathrm{RT}}(u)=\Delta_{\mathrm{RT}}(u)-u \otimes \mathbf{1}-\mathbf{1} \otimes u=\sum_{\substack{V \amalg W=\mathcal{V}(u) \\ W<V, V, W \neq \emptyset}} u_{\left.\right|_{V}} \otimes u_{\left.\right|_{W}},
$$

where the restriction that $V$ and $W$ are nonempty means that $V$ and $W$ give rise to an ordered partition of $\mathcal{V}(u)$ into two blocks. In fact, the iterated restricted coproduct writes in terms of ordered partitions of $\mathcal{V}(u)$ into $n$ blocks:

$$
\widetilde{\Delta}_{\mathrm{RT}}^{n-1}(u)=\left.\sum_{\substack{V_{1} \amalg \cdots \amalg V_{n}=\mathcal{V}(u) \\ V_{n}<\cdots<V_{1}, V_{j} \neq \emptyset}} u\right|_{V_{1}} \otimes \cdots \otimes u_{V_{n}},
$$

and we get the full iterated coproduct $\Delta_{\mathrm{RT}}^{n-1}(u)$ by allowing empty blocks in the formula above. Note that the relation $<$ on subsets of vertices is not transitive. The notation $V_{n}<\cdots<V_{1}$ is to be understood as $V_{i}<V_{j}$ for any $i>j$, with $i, j \in\{1, \ldots, n\}$. 
This Hopf algebra first appeared in the work of A. Dür in 1986 [26], as an incidence Hopf algebra (see section 6). It has been rediscovered and intensively studied by D. Kreimer in 1998 [59], as the Hopf algebra describing the combinatorial part of the BPHZ renormalization procedure of Feynman graphs in a scalar $\varphi^{3}$ quantum field theory. D. Kreimer and A. Connes also proved in [20] that the operator $B_{+}$satisfies the property

$$
\Delta_{\mathrm{RT}}\left(B_{+}\left(t_{1} \cdots t_{n}\right)\right)=B_{+}\left(t_{1} \cdots t_{n}\right) \otimes \mathbf{1}+\left(\operatorname{Id} \otimes B_{+}\right) \circ \Delta_{\mathrm{RT}}\left(t_{1} \cdots t_{n}\right)
$$

for any $t_{1}, \ldots, t_{n} \in \mathcal{T}$. This means that $B_{+}$is a 1 -cocycle in the Hochschild cohomology of $\mathcal{H}_{\mathrm{RT}}$ with values in $\mathcal{H}_{\mathrm{RT}}$, and the couple $\left(\mathcal{H}_{\mathrm{RT}}, B_{+}\right)$is then proved to be universal among commutative Hopf algebras endowed with a 1-cocycle.

The corresponding proalgebraic group $G_{\mathrm{RT}}=\operatorname{Hom}_{\mathrm{CAlg}}\left(\mathcal{H}_{\mathrm{RT}},-\right)$ first appeared in numerical analysis in the work of J. Butcher in 1963 [13, 14, 9], where it was related to the change of Runge-Kutta methods for solving ordinary differential equations. It was then studied by F. Chapoton, who related this group to the pre-Lie operad [18, 19], see section ??.

For any commutative and unital algebra $A$, the group $G_{\mathrm{RT}}(A)$ can be identified with the set of formal series expanded over rooted trees with coefficients in $A$, i.e. the set of maps form the set of nonempty rooted trees to $A$. These $B$-series are by now widely used in the study of approximate solutions of nonlinear differential equations ${ }^{1}[50]$. Such a map extends multiplicatively in a unique way to an $A$-valued character of $\mathcal{H}_{\mathrm{RT}}$. There is an injective morphism of graded Hopf algebras

$$
\Psi: \mathcal{H}_{\mathrm{FdB}} \longleftrightarrow \mathcal{H}_{\mathrm{RT}}
$$

as proven in Paragraph 5.2.2 below. This in turn induces a surjective group homomorphism

$$
G_{\mathrm{RT}}(A) \rightarrow G^{\mathrm{dif}}(A) .
$$

for any commutative algebra $A$.

4.4.2 Feynman graphs and diffeographisms [22] A Feynman graph is a (non planar) graph with a finite number of vertices, together with internal edges (with both ends attached to a vertex) and external edges (with one loose end, the other one being attached to a vertex). A graph is connected if any vertex can be reached from any other vertex by following internal edges. A connected graph is called one-particle irreducible (1PI) if it remains connected after removing any internal edge [21, 71].

In quantum field theory, Feynman graphs are a useful tool to describe some integrals, the amplitudes of the graphs, which compute the expectation value of the events schematically represented by the graphs. The Feynman rules, which recover the amplitude from a given graph, associate to loose ends (viewed as external vertices) some fixed points in the space-time manifold, to (internal) vertices some variable points to be integrated over the space-time manifold, and to edges the propagators of the field theory considered. After a Fourier transform, external edges are associated to fixed external momenta, and internal edges to variable momenta, which are to be integrated. A field theory is prescribed through a Lagrangian, which determines the types of Feynman graphs to be considered: the valence of the internal edges and the possible types of edges. The physically most interesting field theories give rise to Feynman graphs whose amplitude is a divergent integral, and which are therefore called divergent graphs. Physicists have developed some techniques, called renormalization, to extract a meaningful finite contribution from such graphs. A common step of any renormalization process consists in a purely combinatorial manipulation of graphs, in order to take care of possible divergent subgraphs. D. Kreimer proved in [59] that this step can be efficiently described in terms of the Hopf algebra of rooted trees presented in section 4.4.1, where rooted trees encode the hierarchy of divergent subgraphs. A. Connes and D. Kreimer then proved in $[20,21,22]$ that the renormalization Hopf algebra can be defined directly on Feynman graphs.

The so-called Connes-Kreimer Hopf algebra, or Hopf algebra of Feynman graphs, is the free commutative and unital algebra $\mathcal{H}_{\mathrm{FG}}=\mathbb{K}[\mathcal{F}]$ generated by the set $\mathcal{F}$ of connected and 1PI graphs which are superficially divergent, i.e. graphs which are divergent even after having renormalized any divergent subgraph. For any given

\footnotetext{
${ }^{1}$ A B-series is map from the set of rooted trees, including the empty one, to the base field $\mathbb{R}$ or $\mathbb{C}$ of real or complex numbers. A B-series can be identified with an element of the Butcher group if and only if the coefficient of the empty tree is equal to one.
} 
field theory, the superficial divergence of a Feynman graph can be stated as a combinatorial constraint, and implies, for instance, that the number of external legs does not exceed some critical number. The product of 1PI divergent graphs is given by their disjoint union. Hence $\mathcal{H}_{\mathrm{FG}}$ is the vector space generated by superficially divergent locally $1 \mathrm{PI}$ graphs, connected or not, including the empty graph. The coproduct on $\mathcal{H}_{\mathrm{FG}}$ is given on any generator $\Gamma \in \mathcal{F}$ by:

$$
\Delta(\Gamma)=\mathbf{1} \otimes \Gamma+\Gamma \otimes \mathbf{1}+\sum_{\emptyset \subsetneq \gamma \subsetneq \Gamma} \Gamma / \gamma,
$$

where the sum runs over the set of proper and non-empty subgraphs of $\Gamma$ having connected components which are 1PI and superficial divergent. The contracted graph $\Gamma / \gamma$ is obtained by shrinking each connected component of $\gamma$ onto a point $[21,71]$. Note that the condition which ensures that a contracted graph $\Gamma / \gamma$ belongs to $\mathcal{H}_{\mathrm{FG}}$ coincides with the requirement that $\gamma$ only has superficially divergent connected components $[21,2]$.

The counit on $\mathcal{H}_{\mathrm{FG}}$ is given on any generator $\Gamma \in \mathcal{F}$ by $\varepsilon(\Gamma)=0$, and the bialgebra thus obtained is graded by the loop number of the graphs ${ }^{2}$, and connected. Therefore it is automatically a (commutative) Hopf algebra, with antipode defined recursively. The convolution group $G_{\mathrm{FG}}=\operatorname{Hom}_{\mathrm{CAlg}}\left(\mathcal{H}_{\mathrm{FG}},-\right)$ is another example of a prounipotent group, called the group of diffeographisms by Connes and Kreimer in [22].

This affine group scheme plays a key role in the description of the renormalization of quantum fields. Indeed, any splitting $A=A_{-} \oplus A_{+}$of the target algebra into two subalgebras (with $1 \in A_{+}$) yields a Birkhoff decomposition:

$$
G_{\mathrm{FG}}=G_{\mathrm{FG}-} \cdot G_{\mathrm{FG}+}
$$

with $G_{\mathrm{FG}}=\operatorname{Hom}_{\mathrm{CAlg}}\left(\mathcal{H}_{\mathrm{FG}}, A_{ \pm}\right)$. For any $\varphi=\varphi_{-}^{-1} \varphi_{+} \in G_{\mathrm{FG}}$, the renormalized character is the component $\varphi_{+}$, whereas the component $\varphi_{-}$is the counterterm character [21]. The combinatorics underlying this renormalization process relies on a Rota-Baxter property for the projection $A \rightarrow A_{-}$, and applies to any connected graded Hopf algebra in full generality (see [27, 71] and see [28, 29] for a generalization outside the Rota-Baxter framework).

The Hopf algebra of Feynman graphs is naturally endowed with several operators analogous to the grafting operator $B_{+}$defined on the Hopf algebra of rooted trees. In fact, for any primitive graph $\gamma$, i.e. such that $\Delta_{\mathrm{FG}}(\gamma)=\gamma \otimes \mathbf{1}+\mathbf{1} \otimes \gamma$, one can define an insertion operator $\delta \mapsto B_{\gamma}(\delta)$ by summing up all possibilities to insert $\gamma$ inside $\delta$, and taking into account some appropriate symmetry factors (see L. Foissy's notes in the present volume). The insertions are so chosen that, for the resulting graph $\Gamma$, the subgraph $\delta$ appears as the contracted graph $\Gamma / \gamma$. For scalar or fermionic field theories, each operator $B_{\gamma}$ satisfies the same cocycle property (4.4) as $B_{+}$, namely:

$$
\Delta\left(B_{\gamma}(\delta)\right)=B_{\gamma}(\delta) \otimes \mathbf{1}+\left(\operatorname{Id} \otimes B_{\gamma}\right) \circ \Delta(\delta) .
$$

For gauge theories, equation (4.6) takes place in the quotient $\overline{\mathcal{H}}_{\mathrm{FG}}$ of $\mathcal{H}_{\mathrm{FG}}$ by the ideal describing the WardTakahashi identities of the gauge theory [83]. Due to the universal property of the Hopf algebra $\mathcal{H}_{\mathrm{RT}}$ with the operator $B_{+}[35]$, for any primitive graph $\gamma \in \mathcal{H}_{\mathrm{FG}}$ there is a unique graded Hopf algebra morphism

$$
\Phi_{\gamma}: \mathcal{H}_{\mathrm{RT}} \longrightarrow \mathcal{H}_{\mathrm{FG}} \quad \text { (resp. to } \overline{\mathcal{H}}_{\mathrm{FG}} \text { ) }
$$

such that $\Phi_{\gamma} \circ B_{+}=B_{\gamma} \circ \Phi_{\gamma}$. Composing $\Phi_{\gamma}$ with the embedding $\Psi: \mathcal{H}_{\mathrm{FdB}} \longleftrightarrow \mathcal{H}_{\mathrm{RT}}$, we get an injective Hopf algebra morphism

$$
\left.\Phi_{\gamma} \circ \Psi: \mathcal{H}_{\mathrm{FdB}} \hookrightarrow \mathcal{H}_{\mathrm{FG}} \quad \text { (resp. to } \overline{\mathcal{H}}_{\mathrm{FG}}\right)
$$

This embedding was first found by Connes and Kreimer in [22]. The reader will again find a more detailed exposition in L. Foissy's note in the present volume. See also [4] for a concrete application of these Hopf algebra morphisms in quantum field theory. Finally, the map $\Phi_{\gamma} \circ \Psi$ yields a surjective morphism from the group of diffeographisms (resp. a subgroup) to the group of formal diffeomorphisms: for any commutative and unital algebra $A$, there is a canonical projection of groups

$$
G_{\mathrm{FG}}(A) \rightarrow G^{\mathrm{dif}}(A) \quad\left(\text { resp. } \bar{G}_{\mathrm{FG}}(A) \rightarrow G^{\mathrm{dif}}(A)\right) .
$$

\footnotetext{
${ }^{2}$ The loop number is defined by $L(\Gamma)=I(\Gamma)-V(\Gamma)+\left|\pi_{0}(\Gamma)\right|$, where $I(\Gamma)$ stands for the number of internal edges, $V(\Gamma)$ stands for the number of vertices, and $\left|\pi_{0}(\Gamma)\right|$ denotes the number of connected components of the graph.
} 


\subsection{The non-commutative Faà di Bruno Hopf algebra}

Many of the free commutative Hopf algebras related to the Faà di Bruno Hopf algebra admit a natural noncommutative analogue, which in general turns out to be simpler from a combinatorial and from an operadic point of view.

In [35], L. Foissy introduced a non-commutative version of the Hopf algebra $\mathcal{H}_{\mathrm{RT}}$ based on planar rooted trees, and therefore denoted by $\mathcal{H}_{\mathrm{PRT}}$, which has the remarkable property that it is self-dual. The set of generators of $\mathcal{H}_{\mathrm{PRT}}$ as a free noncommutative algebra (planar rooted trees) canonically projects itself onto the set of generators of $\mathcal{H}_{\mathrm{RT}}$ as a free commutative algebra (non-planar rooted trees).

In the case of the Faà di Bruno Hopf algebra, the generators are labeled by natural numbers, and it is also the case for the non-commutative lift described in [15]. This non-commutative Faà di Bruno Hopf algebra is defined as the free associative algebra on the same generators as $\mathcal{H}_{\mathrm{FdB}}$, that is $\mathcal{H}_{\mathrm{FdB}}^{\mathrm{nc}}=\mathbb{K}\left\langle x_{1}, x_{2}, \ldots\right\rangle$, with the usual counit $\varepsilon\left(x_{n}\right)=\delta_{n, 0}$, where $x_{0}=1$, and the coproduct lifted as

$$
\Delta_{\mathrm{FdB}}^{\mathrm{nc}}\left(x_{n}\right)=\sum_{m=0}^{n}\left(\sum_{\substack{k_{0}+\cdots+k_{m}=n-m \\ k_{i} \geq 0}} x_{k_{0}} \cdots x_{k_{m}}\right) \otimes x_{m} .
$$

It is still a graded and connected bialgebra, and the recursive relation for the antipode yields the following non-commutative Lagrange formula (see (4.1) and remark therein), quite involved compared to its commutative counterpart (2.9):

$$
S\left(x_{n}\right)=-x_{n}-\sum_{k=1}^{n-1}(-1)^{k} \sum_{\substack{n_{1}+\cdots+n_{k+1}=n \\ n_{i}>0}} \lambda\left(n_{1}, \ldots, n_{k}\right) x_{n_{1}} \cdots x_{n_{k+1}}
$$

where

$$
\lambda\left(n_{1}, \ldots, n_{k}\right)=\sum_{\substack{m_{1}+\cdots+m_{k}=k \\
m_{1}+\cdots+m_{h} \geq h \text { for } h<k}}\left(\begin{array}{c}
n_{1}+1 \\
m_{1}
\end{array}\right) \cdots\left(\begin{array}{c}
n_{k}+1 \\
m_{k}
\end{array}\right) .
$$

It is then easy to check that $\mathcal{H}_{\mathrm{FdB}}$ coincides with the abelian quotient $\mathcal{H}_{\mathrm{FdB}}^{\mathrm{nc}} /\left[\mathcal{H}_{\mathrm{FdB}}^{\mathrm{nc}}, \mathcal{H}_{\mathrm{FdB}}^{\mathrm{nc}}\right]$, and therefore that the surjective algebra morphism $\pi: \mathcal{H}_{\mathrm{FdB}}^{\mathrm{nc}} \rightarrow \mathcal{H}_{\mathrm{FdB}}$ which identify the generators is a Hopf algebra morphism.

The commutative Hopf algebra $\mathcal{H}_{\text {inv }}$ admits a similar non-commutative lift $\mathcal{H}_{\text {inv }}^{\text {nc }}=\mathbb{K}\left\langle x_{1}, x_{2}, \ldots\right\rangle$, with exactly the same coproduct formula $\Delta_{\text {inv }}\left(x_{n}\right)=\sum_{p=0}^{n} x_{p} \otimes x_{n-p}$. The resulting non-commutative but still co-commutative Hopf algebra is well known under the name of Hopf algebra of non-commutative symmetric functions, the graded dual of which is the Hopf algebra of quasi-symmetric functions [43].

\subsection{Proalgebraic groups on non-commutative algebras}

Proalgebraic groups on non-commutative unital algebras in fact do exist, and should be exhibited by means of free products of algebras. This well-established fact $[6,85,88]$ deserves some explanation, as it may be not so well-known to non-specialists. Although the functor $G^{\text {inv }}$ can be naturally extended to noncommutative algebras along these lines, there seem however to be no means to extend the functor $G^{\text {dif }}$ to non-commutative algebras in this framework: we come back to this point in Paragraph 4.7 below.

4.6.1 Cogroups [6] The free product of associative algebras can be defined as the unique operation $\star$ between associative algebras such that for any two vector spaces $V$ and $W$, there is a canonical algebra isomorphism

$$
T(V) \star T(W) \cong T(V \oplus W)
$$


between associated tensor algebras. For any unital $\mathbb{K}$-algebras $A$ and $B$, the free product $A \star B$ can explicitely be defined as the algebra

$$
A \star B:=T(\bar{A} \oplus \bar{B}) / J
$$

where $\bar{A}=A / \mathbb{K} \cdot 1_{A}$ and $\bar{B}=B / \mathbb{K} \cdot 1_{B}$ are the augmentation ideals, and where $J$ is the ideal of the tensor algebra $T(\bar{A} \oplus \bar{B})$ generated by $\left\{a \otimes a^{\prime}-a a^{\prime}, a, a^{\prime} \in \bar{A}\right\}$ and $\left\{b \otimes b^{\prime}-b b^{\prime}, b, b^{\prime} \in \bar{B}\right\}$. Any element of $A \star B$ is represented by an element of $T(\bar{A} \oplus \bar{B})$, which can be written as a finite sum of terms of the type:

$$
x_{1} \otimes \cdots \otimes x_{r}
$$

where $x_{j}$ is in $\bar{A}$ if and only if $x_{j+1}$ is in $\bar{B}$ for $j=1, \ldots, r-1$, and vice-versa. We write such a term $x_{1} \cdots x_{r}$. The product of two elements in $A \star B$ is given by the concatenation of their representative elements in $T(\bar{A} \oplus \bar{B})$. In particular, there are canonical algebra embeddings $A \cong A \star \mathbb{K} \subset A \star B$ and $B \cong \mathbb{K} \star B \subset A \star B$, and therefore also an embedding of vector spaces

$$
j: A \otimes B \hookrightarrow A \star B,
$$

where $j(A \otimes B)$ contains exactely the terms $x_{1} \otimes x_{2} \in A \star B$ with $x_{1} \in A$ and $x_{2} \in B$. Note however that the map $j$ is not an algebra morphism, because the multiplication in $A \otimes B$ is $\left(m_{A} \otimes m_{B}\right) \circ \tau_{23}$, while the multiplication in $A \star B$ is the concatenation.

The free product is characterized by the following universal property: for any associative unital algebras $A, B, C$ and for any unital algebra morphisms $f: A \rightarrow C$ and $g: B \rightarrow C$, there is a unique algebra morphism $f \mp g: A \star B \rightarrow C$ which extends both $f$ and $g$. The algebra morphism $f \mp g$ is defined by

$$
f \mp g\left(\left[x_{1} \otimes \cdots \otimes x_{r}\right]\right):=h\left(x_{1}\right) \cdots h\left(x_{r}\right),
$$

where $h: \bar{A} \oplus \bar{B} \rightarrow C$ is defined as $h(x)=f(x)$ if $x \in \bar{A}$, and as $h(x)=g(x)$ if $x \in \bar{B}$. This implies that there exists also a canonical projection of vector spaces

$$
\pi: A \star B \rightarrow A \otimes B
$$

which consists in "putting the elements of $\bar{A}$ on the left and the elements of $\bar{B}$ on the right", namely:

$$
\pi\left(\left[x_{1} \otimes \cdots \otimes x_{r}\right]\right)= \begin{cases}x_{1} x_{3} \cdots \otimes x_{2} x_{4} \cdots & \text { if } x_{1} \in \bar{A}, \\ x_{2} x_{4} \cdots \otimes x_{1} x_{3} \cdots & \text { if } x_{1} \in \bar{B} .\end{cases}
$$

It is easily checked that, contrarily to the embedding $j$, the projection $\pi$ is an algebra morphism.

Finally, the free product $\star$ is a bifunctor: given two unital algebra morphisms $f: A \rightarrow A^{\prime}$ and $g: B \rightarrow B^{\prime}$, there exists a straightforward algebra morphism $f \star g: A \star B \rightarrow A^{\prime} \star B^{\prime}$. The multiplication $m_{A}: A \otimes A \rightarrow A$ uniquely extends to an algebra morphism $\widetilde{m}_{A}: A \star A \rightarrow A$ such that $\widetilde{m}_{A}=m_{A} \circ \pi$. We also have $\widetilde{m}_{A}=\operatorname{Id}_{A} \overline{I_{d}}$, and for any unital algebra morphisms $f: A \rightarrow C$ and $g: B \rightarrow C$ we have:

$$
f \star g=\widetilde{m}_{C} \circ(f \star g) .
$$

A cogroup in the category of unital associative $\mathbb{K}$-algebras [30, 6, 5], also called $H$-algebra in [88], is a unital associative $\mathbb{K}$-algebra $H$ together with a "coproduct" $\Delta: H \rightarrow H \star H$, a "counit" $\varepsilon: \mathbb{K} \rightarrow H$ and an "antipode" $S: H \rightarrow H$ such that:

$$
\begin{aligned}
(\mathrm{id} \star \Delta) \circ \Delta & =(\Delta \star \mathrm{id}) \Delta: H \rightarrow H \star H \star H, \\
(\varepsilon \star \mathrm{id}) \circ \Delta & =(\mathrm{id} \star \varepsilon) \circ \Delta=\mathrm{id}, \\
\widetilde{m}_{H} \circ(S \star \mathrm{id}) \circ \Delta & =\widetilde{m}_{H} \circ(\mathrm{id} \star S) \circ \Delta=i \circ \varepsilon .
\end{aligned}
$$

A similar notion has been developed by D. Voiculescu under the name "dual group" in the context of operator algebras [85]. The following proposition, which can be easily derived from the Yoneda lemma (see e.g. [70, Paragraph III.2]), is well-known to experts. However we give a pedestrian proof adapted to our context:

Proposition 1. Let Sets and Alg stand for the category of sets and unital associative algebras respectively. Let 
$H$ be a unital algebra and let $G$ be the functor

$$
\begin{aligned}
\mathrm{Alg} & \longrightarrow \text { Sets } \\
A & \longmapsto G(A)=\operatorname{Hom}_{\mathrm{Alg}}(H, A) .
\end{aligned}
$$

Then $G$ takes its values in the category of groups if and only if the representing object $H$ is a cogroup in the category of unital $\mathbb{K}$-algebras.

Proof. Suppose that $H$ is a cogroup in the category of unital $\mathbb{K}$-algebras, and consider any associative unital algebra $A$. Then we set $g * h=\widetilde{m}_{A} \circ(g \star h) \circ \Delta$ for any unital algebra morphisms $g, h: H \rightarrow A$. The associativity of this product is obvious, as well as the fact that $1_{A} \circ \varepsilon$ is the unit. The fact that $g * h$ is still an algebra morphism comes from the fact that $\widetilde{m}_{A}, g \star h$ and $\Delta$ are. The inverse of any $g$ is given by $g \circ S$, which proves that $\operatorname{Hom}_{\mathrm{Alg}}(H, A)$ is a group.

Conversely, if the functor $G$ takes its values in the category of groupes and is representable as $\operatorname{Hom}_{\mathrm{Alg}}(H,-)$, consider the two embeddings $j_{1}, j_{2}: H \rightarrow H \star H$, which are algebra morphisms, obtained from the two obvious embeddings of $H$ into $T(\bar{H} \oplus \bar{H})$. Then $j_{1} \star j_{2}: H \star H \rightarrow H \star H$ is manifestly equal to the identity. It is then easy to check that the "coproduct" $\Delta$ is given by the product $j_{1} \cdot j_{2}$ in the group $\operatorname{Hom}_{\mathrm{Alg}}(H, H \star H)$. The "counit" is the unit of the group $\operatorname{Hom}_{\mathrm{Alg}}(H, \mathbb{K})$, and the "antipode" is the inverse in the group $\operatorname{Hom}_{\mathrm{Alg}}(H, H)$.

Note that any cogroup $H$ is also a Hopf algebra, by composing the "coproduct" $\Delta$ on the left by the projection $\pi: H \star H \rightarrow H \otimes H$, thus getting a genuine coproduct on $H$. The converse is not true: if $H$ is a Hopf algebra with coproduct $\Delta$, the lift $\widetilde{\Delta}=j \circ \Delta: H \rightarrow H \star H$ is not necessary an algebra morphism, because $j: H \otimes H \hookrightarrow H \star H$ is not. Note also that any cogroup in the category of associative unital algebras is a free algebra [6, Theorem 1.2] or [88].

4.6.2 The cogroup of invertible series One can note that the proalgebraic group $G^{\text {inv }}$ still makes sense on associative unital algebras which are not commutative: for any algebra $A$ of this type, the pointwise multiplication of two formal series of the form $f(t)=1_{A}+\sum_{n \geq 1} f_{n} t^{n}$ with coefficients $f_{n} \in A$ is still an associative operation, and gives rise to a group $G^{\text {inv }}(A)$ which is no longer abelian. One may then wonder about the existence of a functor which associates to any unital algebra $A$ (commutative or not) a convolution group $\operatorname{Hom}_{\mathrm{Alg}}(H, A)$ represented by a non-commutative Hopf algebra $H$, which would reproduce the group $G^{\text {inv }}(A)$ for $H=\mathcal{H}_{\mathrm{inv}}^{\text {nc }}$, and which would give rise to a new group of formal diffeomorphisms for $H=\mathcal{H}_{\mathrm{FdB}}^{\mathrm{nc}}$. Such a functor cannot exist, because if $A$ is a non-commutative algebra, the convolution product of two elements in $\operatorname{Hom}_{\mathrm{Alg}}(H, A)$ has no reason to be an algebra morphism. In the next section we give an explanation of the existence of the non-abelian group $G^{\text {inv }}(A)$ for $A$ non-commutative, but our argument does not justify the existence of the non-commutative Hopf algebra $\mathcal{H}_{\mathrm{FdB}}^{\mathrm{nc}}$ in terms of groups. We come back to this point in Paragraph 4.7 below.

For the non-commutative Hopf algebra $\mathcal{H}_{\mathrm{inv}}^{\text {nc }}=\mathbb{K}\left\langle x_{1}, x_{2}, \ldots\right\rangle$, the free product $\mathcal{H}_{\mathrm{inv}}^{\mathrm{nc}} \star \mathcal{H}_{\mathrm{inv}}^{\mathrm{nc}}$ can be described as the free algebra on two types of variables, one for each copy of $\mathcal{H}_{\mathrm{inv}}^{\mathrm{nc}}$, that is, $\mathcal{H}_{\mathrm{inv}}^{\mathrm{nc}} \star \mathcal{H}_{\mathrm{inv}}^{\mathrm{nc}}=\mathbb{K}\left\langle x_{1}, y_{1}, x_{2}, y_{2} \ldots\right\rangle$. The coproduct $\Delta_{\mathrm{inv}}^{*}: \mathcal{H}_{\mathrm{inv}}^{\mathrm{nc}} \longrightarrow \mathcal{H}_{\mathrm{inv}}^{\text {nc }} \star \mathcal{H}_{\mathrm{inv}}^{\text {nc }}$ defined on generators as

$$
\Delta_{\mathrm{inv}}^{*}\left(x_{n}\right)=\sum_{p=0}^{n} x_{p} y_{n-p} \in \mathcal{H}_{\mathrm{inv}}^{\mathrm{nc}} \otimes \mathcal{H}_{\mathrm{inv}}^{\mathrm{nc}} \subset \mathcal{H}_{\mathrm{inv}}^{\mathrm{nc}} \star \mathcal{H}_{\mathrm{inv}}^{\mathrm{nc}},
$$

is obviously coassociative, and makes $\mathcal{H}_{\text {inv }}^{\text {nc }}$ into a cogroup. Its associated functor $G^{\text {inv }}=\operatorname{Hom}_{\mathrm{Alg}}\left(\mathcal{H}_{\mathrm{inv}}^{\text {nc }},-\right)$ : Alg $\longrightarrow$ Groups then defines the proalgebraic group of invertible series with non-commutative coefficients, namely, for any non-commutative unital associative algebra $A$, the non-abelian group

$$
G^{\mathrm{inv}}(A)=\left\{f(t)=\sum f_{n} t^{n} \mid f_{0}=1\right\} \subset A[[t]]
$$

with pointwise product $(f g)(t)=f(t) g(t)$. The Hopf algebra coproduct $\Delta_{\text {inv }}: \mathcal{H}_{\text {inv }}^{\text {nc }} \longrightarrow \mathcal{H}_{\text {inv }}^{\text {nc }} \otimes \mathcal{H}_{\text {inv }}^{\text {nc }}$ is then just the composition of the cogroup coproduct $\Delta_{\mathrm{inv}}^{*}$ by the canonical projection $\mathcal{H}_{\mathrm{inv}}^{\text {nc }} \star \mathcal{H}_{\mathrm{inv}}^{\text {nc }} \rightarrow \mathcal{H}_{\mathrm{inv}}^{\text {nc }} \otimes \mathcal{H}_{\mathrm{inv}}^{\text {nc }}$. 


\subsection{Open questions about the non-commutative Faà di Bruno Hopf algebra}

Contrarily to invertible series with ordinary multiplication, formal diffeomorphisms $G^{\mathrm{dif}}(A)$ with non-commutative coefficients do not form a group. Indeed, the composition is not associative! As a consequence, the noncommutative Faà di Bruno coproduct $\Delta_{\mathrm{FdB}}^{\mathrm{nc}}$ cannot be derived from a cogroup coproduct $\Delta: \mathcal{H}_{\mathrm{FdB}}^{\mathrm{nc}} \longrightarrow$ $\mathcal{H}_{\mathrm{FdB}}^{\mathrm{nc}} * \mathcal{H}_{\mathrm{FdB}}^{\mathrm{nc}}$ by composing it on the left with the projection $\pi$ onto $\mathcal{H}_{\mathrm{FdB}}^{\mathrm{nc}} \otimes \mathcal{H}_{\mathrm{FdB}}^{\mathrm{nc}}$. One may then ask the following questions:

- Which properties do characterise the set $G^{\mathrm{dif}}(A)$ of formal series with coefficients in a non-commutative algebra $A$, generalizing those of a group, which would allow to define $G^{\text {dif }}$ as a functor represented by $\mathcal{H}_{\mathrm{FdB}}^{\mathrm{nc}}$ ?

- Is there a cogroup coproduct $\Delta: H \longrightarrow H * H$ such that $\pi \circ \Delta: H \rightarrow H \otimes H$ gives $\Delta_{\mathrm{FdB}}$, which would then allow to define a proalgebraic group of formal series $G(A)=\left\{f(t)=t+\sum_{n \geq 2} f_{n} t^{n+1} \mid f_{n} \in A\right\}$ with coefficients in non-commutative algebras $A$, with group law induced by $\Delta$ and therefore different from the composition $(f \circ g)(t)=\sum f_{n} g(t)^{n+1}$ ? If such a group exists, it is probably necessary to rearrange the terms $f_{n} t^{n+1}$ of each series in some non-commutative fashion.

These questions are still open.

\section{Lie algebra interpretation}

\subsection{Vector fields, Witt and Virasoro Lie algebras}

The reader will find a comprehensive treatment of these infinite-dimensional Lie algebras and their representation theory in [55] or in [49]. The Lie algebra Vect $\left(\mathbb{S}^{1}\right)$ of regular vector fields on the circle is, by definition, the Lie algebra of derivations of the ring of Laurent polynomials $\mathbb{R}\left[z, z^{-1}\right]$. It can be seen as the real vector space

$$
\operatorname{Vect}\left(\mathbb{S}^{1}\right)=\operatorname{Span}_{\mathbb{R}}\left\{e_{n}=\frac{1}{2 i \pi} e^{2 i \pi n t} \frac{\mathrm{d}}{\mathrm{d} t}, n \in \mathbb{Z}\right\},
$$

where $z=e^{2 i \pi t}$. The Lie bracket is given by:

$$
\left[e_{m}, e_{n}\right]=(n-m) e_{n+m} .
$$

The same Lie algebra can be considered over any base field $\mathbb{K}$, and in the case of a field of characteristic $p>0$ it was first introduced by E. Witt in a Hamburg Seminar in 1939, and reported by H. Zassenhaus in [87], with generators restricted to $1 \leq n \leq p-2$.

By extension, the complex Lie algebra Vect $\left(\mathbb{S}^{1}\right)_{\mathbb{C}}$, which can be understood as the Lie algebra of polynomial complex functions on the circle, is now called the Witt Lie algebra and denoted by $\mathcal{W}(\mathbb{C})$. Its one-dimensional central extension is the so-called Virasoro Lie algebra, first found in positive characteristic by R. E. Block in 1966 [7], and then in characteristic zero by I.M. Gelfand and D.B. Fuks in 1968 [41]. These two Lie algebras describe the infinitesimal conformal transformations in two real dimensions (with or without anomaly), and play a central role in conformal field theory and in string theory [49]. Two-dimensional conformal field theory is deeply related to the theory of vertex operator algebras (see e.g. the introduction of [38]).

The Lie algebra of formal vector fields on a line was defined by E. Cartan as early as 1909 [16]:

$$
\mathcal{W}_{+}(\mathbb{K})=\operatorname{Span}_{\mathbb{K}}\left\{e_{n}=t^{n+1} \frac{\mathrm{d}}{\mathrm{d} t}, n \geq-1\right\} 3^{3}
$$

When $\mathbb{K}=\mathbb{C}, \mathcal{W}_{+}(\mathbb{C})$ gives the vector fields on $\mathbb{S}^{1}$ which extend holomorphically to the unit disk. In his book, Fuks considers as well the following Lie subalgebras of $\mathcal{W}_{+}(\mathbb{K})$ :

$$
L_{k}=\operatorname{Span}_{\mathbb{K}}\left\{e_{n}=t^{n+1} \frac{\mathrm{d}}{\mathrm{d} t}, n \geq k\right\},
$$

\footnotetext{
${ }^{3}$ This Lie algebra is denoted $W_{1}(\mathbb{K})$ by D.B. Fuks in [40], who considers also the Lie algebras of formal vector fields on any $n$-dimensional space, $W_{n}(\mathbb{K})$.
} 
satisfying the properties

$$
\mathcal{W}_{+}(\mathbb{K})=L_{-1} \supset L_{0} \supset L_{1} \supset \cdots, \quad \text { and } \quad\left[L_{m}, L_{n}\right] \subset L_{m+n} \quad \text { for } m, n \geq 0 .
$$

In particular, the Lie algebra $L_{1}$ is pronilpotent [17]. We show in the next section that $L_{1}$ is the Lie algebra of the group of formal diffeomorphisms $G^{\text {dif }}(\mathbb{K})$.

\subsection{Lie algebra of algebraic and proalgebraic groups}

Let $G_{H}$ be a proalgebraic group represented by a commutative Hopf algebra $H$ over a field $\mathbb{K}$. For any commutative unital $\mathbb{K}$-algebra $A$, an $A$-valued infinitesimal character of $H$, or $A$-valued derivation on $H$ [20, 71], is a linear map $\alpha: H \rightarrow A$ such that, for any $x, y \in H$, the following relation holds:

$$
\alpha(x y)=\alpha(x) e_{A}(y)+e_{A}(x) \alpha(y),
$$

where $e_{A}=i_{A} \circ \varepsilon$. Since $e_{A}\left(1_{H}\right)=1_{A}$, this implies that $\alpha\left(1_{H}\right)=0$. Then, it is easily checked that the set $\mathfrak{g}_{H}(A)=\operatorname{Der}(H, A)$ of $A$-valued derivations on $H$ is a Lie algebra, with bracket given by

$$
[\alpha, \beta]:=\alpha * \beta-\beta * \alpha,
$$

for any $\alpha, \beta \in \mathfrak{g}_{H}(A)$. Moreover, the map $A \mapsto \mathfrak{g}_{H}(A)$ is clearly a functor $\mathfrak{g}_{H}$ from the category of commutative unital algebras into the category of Lie algebras. If $H$ is a finitely generated algebra (resp. inductive limit of finitely generated algebras), the functor $\mathfrak{g}_{H}$ is by definition the Lie algebra of the algebraic (resp. proalgebraic) group $G_{H}$.

Proposition 2. Let $G_{H}$ be a prounipotent group, with Lie algebra $\mathfrak{g}_{H}$, and suppose that the base field $\mathbb{K}$ is of characteristic zero. For any commutative unital algebra $A$, the exponential map

$$
\begin{aligned}
\exp : \mathfrak{g}_{H}(A) & \longrightarrow G_{H}(A) \\
\alpha & \longmapsto \exp (\alpha):=e_{A}+\sum_{n \geq 1} \frac{\alpha^{* n}}{n !}
\end{aligned}
$$

is a bijection, and we have $\exp (\alpha) * \exp (\beta)=\exp (\alpha+\beta)$ if $[\alpha, \beta]=0$. The inverse of the exponential map is the logarithm, defined by

$$
\begin{aligned}
\log : G_{H}(A) & \longrightarrow \mathfrak{g}_{H}(A) \\
\varphi=e_{A}+\gamma & \longmapsto \log (\varphi)=\sum_{n \geq 1} \frac{(-1)^{n-1} \gamma^{* n}}{n} .
\end{aligned}
$$

Proof. Let $m$ be a positive integer. For any $x \in H^{m}$ we have:

$$
\Delta(x)=x \otimes 1_{H}+1_{H} \otimes x+\sum_{(x)} x^{\prime} \otimes x^{\prime \prime},
$$

where $x^{\prime}, x " \in H^{m-1}$. As a consequence, for any linear map $\alpha: H \rightarrow A$ such that $\alpha\left(1_{H}\right)=0$, we have $\alpha^{* n}(x)=0$ for any $n \geq m+1$. This applies in particular when $\alpha$ is an infinitesimal character. Hence the exponential of $\alpha$ is well-defined, as the sum which defines it is locally finite. The same argument applies to the logarithm. The fact that the exponential of an infinitesimal character is a character is checked by direct inspection, as well as the last assertion. Finally let us consider any character $\varphi \in G_{H}(A)$. The powers $\varphi^{* m}$ are also characters for any positive integer $m$. Now let us define for any $\lambda \in \mathbb{K}$ :

$$
\varphi^{* \lambda}:=\exp (\lambda \log (\varphi))
$$

For any $x, y \in H$, the expression $\varphi^{* \lambda}(x) \varphi^{* \lambda}(y)-\varphi^{* \lambda}(x y)$ is polynomial in $\lambda$ and vanishes at any positive integer, hence vanishes identically. It follows that $\varphi^{* \lambda}$ is a character for any $\lambda \in \mathbb{K}$. Differentiating with respect to $\lambda$ at $\lambda=0$ immediately gives the infinitesimal character equation for $\log (\varphi)$. A standard direct computation then shows that the logarithm and the exponential are mutually inverse.

Suppose now that the proalgebraic group $G_{H}$ is represented by a graded Hopf algebra $H=\oplus_{n \geq 0} H_{n}$, such that each graded component $H_{n}$ is finite-dimensional. Then one can define the graded dual Hopf algebra 
$H^{*}:=\bigoplus_{n} H_{n}^{*}$ with the operations given in section 4.1. Since $H$ is commutative, its dual $H^{*}$ is a cocommutative Hopf algebra. By the Cartier-Milnor-Moore theorem, then, $H^{*}$ is the universal enveloping algebra of the Lie algebra formed by its primitive elements,

$$
\operatorname{Prim} H^{*}=\left\{\alpha: H \longrightarrow \mathbb{K} \text { linear, } m^{*}(\alpha)=\alpha \otimes \varepsilon+\varepsilon \otimes \alpha\right\} .
$$

Applying the definition of $m^{*}$ one sees immediately that such primitive elements are $\mathbb{K}$-valued infinitesimal characters on $H$, and that Prim $H^{*}$ is a Lie subalgebra of the Lie algebra $\mathfrak{g}_{H}(\mathbb{K})$. In fact, $\mathfrak{g}_{H}(\mathbb{K})$ is the completion of Prim $H^{*}$.

5.2.1 Lie algebra for the Faà di Bruno Hopf algebra As we saw, the group of formal diffeomorphisms is a prounipotent group represented by the Faà di Bruno Hopf algebra $\mathcal{H}_{\mathrm{FdB}}=\mathbb{K}\left[x_{1}, x_{2}, \ldots\right]$. This algebra is the free commutative algebra (i.e. the symmetric algebra) on the vector space spanned by the graded variables $x_{n}$, with degree $\left|x_{n}\right|=n$. Therefore it is graded, and its graded dual space $\mathcal{H}_{\mathrm{FdB}}^{*}$ is the universal enveloping algebra of a Lie algebra that we now describe.

The dual Hopf algebra $\mathcal{H}_{\mathrm{FdB}}^{*}$ is the cofree graded cocommutative coalgebra (the so-called symmetric coalgebra) on the vector space spanned by the dual elements of the variables $x_{n}$, that we denote by $e_{n}$. To be more precise, $\mathcal{H}_{\mathrm{FdB}}^{*}$ is isomorphic, as a graded vector space, to the symmetric algebra $S(V)$ where $V$ is the span of linear maps $e_{n}: \mathcal{H}_{\mathrm{FdB}} \longrightarrow \mathbb{K}$ such that $e_{n}\left(x_{m}\right)=\delta_{n m}$. The grading is induced by the grading on $V$ given by $\left|e_{n}\right|=-n$.

Elements of $V$ are clearly infinitesimal characters of $\mathcal{H}_{\mathrm{FdB}}$, i.e. elements of $\mathfrak{g}_{\mathcal{H}_{\mathrm{FdB}}}(\mathbb{K})$. The Hopf algebra structure on $\mathcal{H}_{\mathrm{FdB}}^{*}$ is induced by that of $\mathcal{H}_{\mathrm{FdB}}$ : the free commutative product on $\mathcal{H}_{\mathrm{FdB}}$ gives the unshuffle coproduct [75] on $\mathcal{H}_{\mathrm{FdB}}^{*}$, defined by:

$$
\Delta\left(e_{i_{1}} \cdots e_{i_{k}}\right)=\sum_{I \amalg J=\{1, \ldots k\}} e_{I} \otimes e_{J},
$$

where $I$ and $J$ are complementary parts of $\{1, \ldots, k\}$ and $e_{I}$ (resp. $e_{J}$ ) stands for the symmetric product of $e_{i_{j}}$ 's with $j \in I$ (resp. $j \in J$ ). The Faà di Bruno coproduct on $\mathcal{H}_{\mathrm{FdB}}$ gives the (noncommutative) multiplication on $\mathcal{H}_{\mathrm{FdB}}^{*}$ (of course different from the symmetric product we used just above). It is easy to check that the generators $e_{n}$ are the only primitive elements with respect to the unshuffle coproduct, therefore

$$
\operatorname{Prim} \mathcal{H}_{\mathrm{FdB}}^{*}=\operatorname{Span}_{\mathbb{K}}\left\{e_{1}, e_{2}, \ldots\right\} \text {. }
$$

On the other hand, the multiplication induced by $\Delta_{\mathrm{FdB}}$ on $\mathcal{H}_{\mathrm{FdB}}^{*}$ coincides with the convolution product when it is restricted to $\mathcal{L}(H, \mathbb{K})$. By direct computations (or see [12] about $\left(\mathcal{H}_{\mathrm{FdB}}^{\mathrm{nc}}\right)^{o p}$ ), we then obtain, for any $p, q \in \mathbb{N}-\{0\}:$

$$
\left[e_{p}, e_{q}\right]:=e_{p} * e_{q}-e_{q} * e_{p}=(q-p) e_{p+q} .
$$

This means that $L_{1}$ is the Lie algebra of the group $G^{\mathrm{dif}}(\mathbb{K})^{o p}$.

Finally note that the convolution product restricted to the generators of $L_{1}$ can be written as

$$
e_{p} * e_{q}=e_{p} \triangleright e_{q}+\varphi
$$

where $\varphi$ is any element of $L_{1}$ which, seen as a linear map on $\mathcal{H}_{\mathrm{FdB}}$, vanishes on the $x_{n}$ 's for any $n \in \mathbb{N}$, and where we set

$$
e_{p} \triangleright e_{q}:=(q+1) e_{p+q} .
$$

The binary operation $\triangleright$ is a left pre-Lie product $[42,84]$, which means that it satisfies the condition

$$
e_{p} \triangleright\left(e_{q} \triangleright e_{r}\right)-\left(e_{p} \triangleright e_{q}\right) \triangleright e_{r}=e_{q} \triangleright\left(e_{p} \triangleright e_{r}\right)-\left(e_{q} \triangleright e_{p}\right) \triangleright e_{r},
$$

for any $p, q, r>0$. Such products are discussed in section 6.2.2. Going further, there is even a family $\left(\triangleright_{\lambda}\right)_{\lambda \in \mathbb{K}}$ of left pre-Lie products on $L_{1}$, given by $e_{p} \triangleright_{\lambda} e_{q}=(p+\lambda) e_{p+q}$, whose antisymmetrization gives back the Lie bracket 5.1. See L. Foissy's notes in the present volume for more details. 
5.2.2 Lie algebra for the Hopf algebra of rooted trees The graded dual $\mathcal{H}_{\mathrm{RT}}^{*}$ of the Hopf algebra of rooted trees is called Grossman-Larson Hopf algebra, after the first authors who considered it [47]. Being a cocommutative Hopf algebra, it is the enveloping algebra of a Lie algebra that we now describe.

The Hopf algebra $\mathcal{H}_{\mathrm{RT}}$ is the free commutative algebra on the vector space spanned by rooted trees. It is a graded Hopf algebra, if we consider the trees to be graded by the number of their vertices. A basis of $\mathcal{H}_{\mathrm{RT}}$ is given by monomials of trees, i.e. forests $(s)$. Denote by $\left(\delta_{s}\right)$ the dual basis in $\mathcal{H}_{\mathrm{RT}}^{*}$. The correspondence $\delta: s \mapsto \delta_{s}$ extends linearly to a unique vector space isomorphism from $\mathcal{H}_{\mathrm{RT}}$ onto $\mathcal{H}_{\mathrm{RT}}^{*}$. For any tree $t$, the corresponding $\delta_{t}$ is a primitive element of $\mathcal{H}_{\mathrm{RT}}^{*}$. In particular, it is an infinitesimal character of $\mathcal{H}_{\mathrm{RT}}$, i.e. an element of $\mathfrak{g}_{\mathcal{H}_{\mathrm{RT}}}(\mathbb{K})$. The Lie algebra of the group $G_{\mathrm{RT}}(\mathbb{K})$ is then the vector space

$$
\operatorname{Lie}\left(G_{\mathrm{RT}}(\mathbb{K})\right)=\operatorname{Span}_{\mathbb{K}}\left\{\delta_{t}, t \in \mathcal{T}\right\}
$$

with Lie bracket

$$
\left[\delta_{t}, \delta_{u}\right]:=\delta_{t} * \delta_{u}-\delta_{u} * \delta_{t}=\delta_{t \curvearrowright u-u \curvearrowright t},
$$

where $t \curvearrowright u$ is the tree obtained by grafting $t$ on $u$ according to the rule

$$
t \curvearrowright u=\sum_{T \in \mathcal{T}} N^{\prime}(t, u, T) T,
$$

where $N^{\prime}(t, u, T)$ is the number of partitions $\mathcal{V}(T)=V \amalg W$ of the set of vertices of the tree $T$ into two subsets with $W<V$, and such that $T_{\left.\right|_{V}}=v$ and $T_{\left.\right|_{W}}=u$. Another normalization is often employed to describe this Lie algebra. Consider in $\mathcal{H}_{\mathrm{RT}}^{*}$ the normalized dual basis $\widetilde{\delta}_{t}=\sigma(t) \delta_{t}$, where $\sigma(t)=\mid$ Aut $t \mid$ stands for the symmetry factor of $t$. Then

$$
\operatorname{Lie}\left(G_{\mathrm{RT}}(\mathbb{K})\right) \cong \operatorname{Span}_{\mathbb{K}}\left(\widetilde{\delta}_{t}, t \in \mathcal{T}\right)
$$

with Lie bracket

$$
\left[\widetilde{\delta}_{t}, \widetilde{\delta}_{u}\right]:=\widetilde{\delta}_{t} * \widetilde{\delta}_{u}-\widetilde{\delta}_{u} * \widetilde{\delta}_{t}=\widetilde{\delta}_{t \rightarrow u-u \rightarrow t},
$$

given in terms of the grafting operation

$$
t \rightarrow u=\sum_{T \in \mathcal{T}} M^{\prime}(t, u, T) T,
$$

where the factor $M^{\prime}(t, u, T)=\frac{\sigma(t) \sigma(u)}{\sigma(T)} N^{\prime}(t, u, T)$ can be interpreted as the number of ways to graft the tree $t$ on the tree $u$ in order to get the tree $T$. Otherwise said, $t \rightarrow u$ is the sum of the trees obtained by grafting $t$ on $u$ at any vertex $v$ :

$$
t \rightarrow u=\sum_{v \in \mathcal{V}(u)} t \rightarrow_{v} u
$$

The interesting aspect of the last presentation of $\operatorname{Lie}\left(G_{\mathrm{RT}}(\mathbb{K})\right)$ is that the binary operation $\rightarrow$ on trees is a left pre-Lie product, i.e., for any trees $s, t, u$, we have:

$$
s \rightarrow(t \rightarrow u)-(s \rightarrow t) \rightarrow u=t \rightarrow(s \rightarrow u)-(t \rightarrow s) \rightarrow u
$$

This identity is easily checked by remarking that the left-hand side is obtained by summing up all the trees obtained by grafting $s$ and $t$ on two (distinct or equal) vertices of $u$. This procedure is obviously symmetric in $s$ and $t$. Moreover, it has been shown by F. Chapoton and M. Livernet [19] that the vector space spanned by the rooted trees $\mathcal{T}$, endowed with the binary product $\rightarrow$, is in fact the free pre-Lie algebra with one generator.

Let us consider the pre-Lie algebra $\left(L_{1}, \triangleright\right)$ with basis $\left(e_{n}\right)_{n \geq 1}$ introduced in Paragraph 5.2.1. Using the universal property of the free pre-Lie algebra, there is a unique pre-Lie algebra morphism

$$
\phi:(\mathcal{T}, \rightarrow) \longrightarrow(\mathcal{W}, \triangleright)
$$

such that $\phi(\bullet)=e_{1}$. It is a fortiori a Lie algebra morphism, and it is obviously surjective: indeed $e_{n}$ is the image of $\frac{1}{n !} \ell_{n}$, where $\ell_{n}$ is the ladder tree with $n$ vertices. It extends to a surjective Hopf algebra morphism between the corresponding enveloping algebras. Finally, the transposed map gives rise to the Hopf algebra embedding (4.5), namely $\Psi: \mathcal{H}_{\mathrm{FdB}} \longleftrightarrow \mathcal{H}_{\mathrm{RT}}$, of section 4.4.1. 


\section{Combinatorial interpretation}

\subsection{Incidence Hopf algebras}

Incidence Hopf algebras are Hopf algebras built from suitable families of partially ordered sets. They have been elaborated by W. R. Schmitt in 1994 [78], following the track opened by S. A. Joni and G.-C. Rota when they defined incidence algebras and coalgebras ([77, 54], see also [79]). They form a large family of Hopf algebras, which includes those on trees and the Faà di Bruno one. We quickly describe here the subfamily of "standard reduced" incidence Hopf algebras, which are always commutative.

A poset is a partially ordered set $P$, with order relation denoted by $\leq$ For any $x, y \in P$, the interval $[x, y]$ is the subset of $P$ formed by the elements $z$ such that $x \leq z \leq y$. Let $\mathcal{P}$ be a family of finite posets $P$ such that there exists a unique minimal element $0_{P}$ and a unique maximal element $1_{P}$ in $P$ (hence $P$ coincides with the interval $\left.P=\left[0_{P}, 1_{P}\right]\right)$. This family is called interval closed if for any poset $P \in \mathcal{P}$ and for any $x \leq y \in P$, the interval $[x, y]$ is an element of $\mathcal{P}$. Let $\overline{\mathcal{P}}$ be the quotient $\mathcal{P} / \sim$, where $P \sim Q$ if and only if $P$ and $Q$ are isomorphic as posets ${ }^{4}$. The equivalence class of any poset $P \in \mathcal{P}$ is denoted by $\bar{P}$ (notation borrowed from [31]). The standard reduced incidence coalgebra of the family of posets $\mathcal{P}$ is the $\mathbb{K}$-vector space freely generated by $\overline{\mathcal{P}}$, with coproduct given by

$$
\Delta[P]=\sum_{x \in P} \overline{\left[0_{P}, x\right]} \otimes \overline{\left[x, 1_{P}\right]},
$$

and counit given by $\varepsilon(\overline{\{*\}})=1$ and $\varepsilon(\bar{P})=0$ if $P$ contains two elements or more.

Given two posets $P$ and $Q$, the direct product $P \times Q$ is the set-theoretic cartesian product of the two posets, with partial order given by $(p, q) \leq\left(p^{\prime}, q^{\prime}\right)$ if and only if $p \leq p^{\prime}$ and $q \leq q^{\prime}$. A family of finite posets $\mathcal{P}$ is called hereditary if the product $P \times Q$ belongs to $\mathcal{P}$ whenever $P, Q \in \mathcal{P}$. The quotient $\overline{\mathcal{P}}$ is then a commutative semigroup generated by the set $\overline{\mathcal{P}_{0}}$ of classes of indecomposable posets, i.e. posets $R \in \mathcal{P}$ such that for any $P, Q \in \mathcal{P}$ of cardinality $\geq 2, P \times Q$ is not isomorphic to $R$. The commutativity comes from the obvious isomorphism $P \times Q \sim Q \times P$ for any $P, Q \in \mathcal{P}$. The unit element $\mathbf{1}$ is the class of any poset with only one element.

Proposition 3. [78, Theorem 4.1] If $\mathcal{P}$ is a hereditary family of finite posets, the standard reduced coalgebra $\mathcal{H}(\mathcal{P})$ of $\mathcal{P}$ is a Hopf algebra.

Proof. The semigroup product extends bilinearly to an associative product on $\mathcal{H}(\mathcal{P})$. The compatibility of the coproduct with this product is obvious. The unit is a coalgebra morphism and the counit is an algebra morphism. The existence of the antipode comes from the fact that for any poset $P \in \mathcal{P}$ of cardinal, say, $n$, we obviously have:

$$
\Delta(\bar{P})=\bar{P} \otimes \mathbf{1}+\mathbf{1} \otimes \bar{P}+\sum_{(\bar{P})} \overline{P^{\prime}} \otimes \overline{P^{\prime \prime}},
$$

where $P^{\prime}$ and $P "$ contain strictly less than $n$ elements (note that the fact that $P$ is the interval $\left[0_{P}, 1_{P}\right]$ is crucial here). Considering the reduced coproduct $\widetilde{\Delta}(\bar{P})=\Delta(\bar{P})-\bar{P} \otimes \mathbf{1}-\mathbf{1} \otimes \bar{P}$, the iterated reduced coproduct $\widetilde{\Delta}^{m}(\bar{P})$ therefore vanishes for $m>n$. It is well-known (see e.g. [71]) that this conilpotence property allows us to define the convolution inverse of the identity, and even of any linear map $\varphi: \mathcal{H}(\mathcal{P}) \rightarrow \mathcal{H}(\mathcal{P})$ with $\varphi(\mathbf{1})=\mathbf{1}$.

Many of the Hopf algebras encountered so far are incidence Hopf algebras. We give three examples, all of them borrowed from [78].

6.1.1 The binomial and the divided power Hopf algebras Let $\mathcal{B}$ be the family of finite boolean algebras. An element of $\mathcal{B}$ is any poset isomorphic to the set $\mathcal{P}(A)$ of all subsets of a finite set $A$. The partial order on $\mathcal{P}(A)$ is given by the inclusion. If $B$ and $C$ are two subsets of $A$ with $B \subset C$, the interval $[B, C]$ in $\mathcal{P}(A)$ is isomorphic to $\mathcal{P}(C \backslash B)$, hence $\mathcal{B}$ is interval-closed. Moreover the obvious property:

$$
\mathcal{P}(A) \times \mathcal{P}(B) \sim \mathcal{P}(A \amalg B)
$$

\footnotetext{
${ }^{4} \mathrm{~W}$. Schmitt allows more general equivalence relations, called order-compatible relations.
} 
implies that $\mathcal{B}$ is hereditary. The incidence Hopf algebra $\mathcal{H}(\mathcal{B})$ is the so-called binomial Hopf algebra, because of the expression of the coproduct on generators. In fact, as a vector space $\mathcal{H}(\mathcal{B})$ is clearly spanned by $\left\{x_{0}, x_{1}, x_{2}, \ldots\right\}$, where $x_{n}$ stands for the isomorphism class of $\mathcal{P}(\{1, \ldots, n\})$. The product is obviously given by $x_{m} x_{n}=x_{m+n}$, the unit is $\mathbf{1}=x_{0}$, the counit is given by $\varepsilon\left(x_{n}\right)=0$ for $n \geq 1$, and the coproduct is entirely defined by $\Delta\left(x_{1}\right)=x_{1} \otimes \mathbf{1}+\mathbf{1} \otimes x_{1}$. Explicitly we have:

$$
\Delta\left(x_{n}\right)=\sum_{k=0}^{n}\left(\begin{array}{l}
n \\
k
\end{array}\right) x_{k} \otimes x_{n-k} .
$$

The graded dual Hopf algebra $\mathcal{H}(\mathcal{B})^{*}$ is known as the divided power algebra: it can be represented as the vector space $\mathbb{K}\left\{y_{0}, y_{1}, y_{2}, \ldots\right\}$ with multiplication

$$
y_{m} \star y_{n}=\left(\begin{array}{c}
m+n \\
m
\end{array}\right) y_{m+n}
$$

and unit $\mathbf{1}=y_{0}$. The counit is $\varepsilon\left(y_{n}\right)=0$ if $n>0$, and the coproduct is given by

$$
\Delta\left(y_{n}\right)=\sum_{p+q=n} y_{p} \otimes y_{q} .
$$

6.1.2 The Faà di Bruno Hopf algebra Let $\mathcal{S P}$ be the family of posets isomorphic to the set $\mathcal{S P}(A)$ of all partitions of some nonempty finite set $A$. The partial order on set partitions is given by refinement. We denote by $0_{A}$ or 0 the partition by singletons, and by $1_{A}$ or 1 the partition with only one block. Let $\mathcal{Q}$ be the family of posets isomorphic to the cartesian product of a finite number of elements in $\mathcal{S P}$. If $S$ and $T$ are two partitions of a finite set $A$ with $S \leq T$ (i.e. $S$ is finer than $T$ ), the partition $S$ restricts to a partition of any block of $T$. Denoting by $W / S$ the set of those blocks of $S$ which are included in some block $W$ of $T$, any partition $U$ such that $S \leq U \leq T$ yields a partition of the set $W / S$ for any block $W$ of $T$. This in turn yields the following obvious poset isomorphism:

$$
[S, T] \sim \prod_{W \in A / T} \mathcal{S P}(W / S)
$$

This shows that $\mathcal{Q}$ is interval closed (and hereditary by definition).

Proposition 4. [78, Example 14.1] The incidence Hopf algebra $\mathcal{H}(\mathcal{Q})$ is isomorphic to the Faà di Bruno Hopf algebra.

Proof. Denote by $X_{n}$ the isomorphism class of $\mathcal{S P}(\{1, \ldots, n+1\})$. Note that $X_{0}$ is the unit of the Hopf algebra. In view of (6.2), we have:

$$
\begin{aligned}
\Delta\left(X_{n}\right) & =\sum_{S \in \mathcal{S P}(\{1, \ldots, n+1\})} \overline{[0, S]} \otimes \overline{[S, 1]} \\
& =\sum_{S \in \mathcal{S P}(\{1, \ldots, n+1\})}\left(\prod_{W \in\{1, \ldots, n+1\} / S} \overline{\mathcal{S P}(W)}\right) \otimes \overline{\mathcal{S P}(\{1, \ldots, n+1\} / S)} .
\end{aligned}
$$

The coefficient in front of $X_{1}^{k_{1}} \cdots X_{n}^{k_{n}} \otimes X_{m}$ in (6.3) is equal to the number of partitions of $\{1, \cdots, n+1\}$ with $k_{j}$ blocks of size $j+1$ (for $j=1$ to $n$ ), $m+1$ blocks altogether, and $k_{0}=m+1-k_{1}-\cdots-k_{n}$ blocks of size 1 . By definition of the Bell polynomials in terms of partitions, we have then:

$$
\Delta\left(X_{n}\right)=\sum_{m=0}^{n} B_{m+1, n+1}\left(X_{0}, X_{1}, X_{2}, \ldots\right) \otimes X_{m},
$$

which in turn gives $(4.2)$ with $x_{j}:=\frac{1}{(j+1) !} X_{j}$.

6.1.3 The Hopf algebra of rooted trees Let $P$ be a finite poset not assumed tobe isomorphic to an interval. As an example, we can take as poset $P$ the vertex set $\mathcal{V}(F)$ of a rooted forest $F$, in which $v \leq w$ if and only if there is a path from one root to $w$ through $v$. An order ideal (or initial segment) in $P$ is a subset $I$ of $P$ such 
that for any $w \in I$, if $v \leq w$, then $v \in I$. For a rooted forest $F$, an initial segment in $\mathcal{V}(F)$ is a subforest such that any connected component of it contains a root of $F$. For any finite poset $P$, we denote by $J(P)$ the poset of all initial segments of $P$, ordered by inclusion [78, Paragraph 16]. The minimal element $0_{J(P)}$ is the empty set, and the maximal element $1_{J(P)}$ is $P$. For two finite posets $P$ and $Q$ one obviously has:

$$
J(P \amalg Q) \sim J(P) \times J(Q) .
$$

The isomrphism class of a poset $P$ is uniquely determined by the isomorphism class of the poset $J(P)$. To see this, consider two posets $P$ and $Q$, and suppose there is an isomorphism $\Phi: J(P) \rightarrow J(Q)$. For any $x \in P$, consider the initial segment $P_{\leq x}:=\{y \in P, y \leq x\}$. It has $x$ as unique maximal element. Now, $\Phi\left(P_{\leq x}\right)$ has a unique maximal element which we denote by $\varphi(x)$, and it is not hard to see that the map $\varphi: P \rightarrow Q$ thus constructed is a poset isomorphism.

For a poset $P$ and two initial segments $I_{1} \subset I_{2} \subset P$ with $I_{1}$ fixed, the correspondence $I_{2} \mapsto I_{2} \backslash I_{1}$ defines a poset isomorphism:

$$
\left[I_{1}, I_{2}\right] \subset J(P) \longrightarrow J\left(I_{2} \backslash I_{1}\right) .
$$

Differences $Q=I_{2} \backslash I_{1}$ are convex subsets of $P$, i.e. such that for any $x, y \in Q$, we have $[x, y] \leq Q$. Conversely, any convex subset $Q \subset P$ can be written as a difference $P_{\leq Q} \backslash P_{<Q}$ of two unique initial segments:

$$
\begin{aligned}
& P_{\leq Q}:=\{x \in P, \exists y \in Q, x \leq y\}, \\
& P_{<Q}:=\{x \in P, \forall y \in Q, x<y\} .
\end{aligned}
$$

Now let $\mathcal{F}$ be a family of finite posets which is closed by disjoint unions and such that for any poset $P \in \mathcal{F}$, convex subsets of $P$ also belong to $\mathcal{F}$. Then the corresponding family:

$$
J(\mathcal{F}):=\{J(P), P \in \mathcal{F}\}
$$

is hereditary by virtue of isomorphisms (6.5) and (6.6).

Proposition 5. The family $\mathcal{F}$ of rooted forests is stable by taking disjoint unions and convex subposets, and the associated incidence Hopf algebra $\mathcal{H}(J(\mathcal{F}))$ is isomorphic to $\mathcal{H}_{\mathrm{RT}}$.

Proof. Via the isomorphism $\Phi$ defined above, the vector space $\mathcal{H}(J(\mathcal{F}))$ can be identified with the vector space freely generated be the rooted forests. By (6.5), the product is then given by disjoint union, and the coproduct writes:

$$
\Delta(P)=\sum_{I \in J(P)}(P \backslash I) \otimes I,
$$

which is just the coproduct (4.3) modulo flipping the terms (we have denoted by the same letter a forest and its underlying poset). The counit is given by $\varepsilon(\mathbf{1})=1$ and $\varepsilon(P)=0$ for any nontrivial forest $P$.

This example can be extended, still in the context of incidence Hopf algebras, to oriented cycle-free graphs [72]. Hopf algebras of Feynman graphs do not enter strictly speaking into this framework (see however [78, Paragraph 14] about Hopf algebras of simple 3-connected graphs). For other examples of incidence coalgebras and Hopf algebras, see [54, 78].

\subsection{Combinatorial Hopf algebras}

The Faà di Bruno Hopf algebra has some important properties also satisfied by almost all examples of graded Hopf algebras we have encountered so far (see [54]). These properties are:

- to be connected,

- to be free or free commutative (or, dually, cofree or cofree cocommutative),

- to have an explicit basis, 
- to have the "right-sided property":

$$
\Delta\left(x_{n}\right)=\sum\left(\text { polynomial in } x_{k}\right) \otimes x_{m}
$$

or its left analogue.

Hopf algebras of this type have been called combinatorial Hopf algebras ${ }^{5}$ by J.-L. Loday and M. Ronco in [67]. In particular, any combinatorial Hopf algebra fits into one of the four types below:

$$
\begin{gathered}
\left(T^{a}(V), \otimes, \Delta ?\right) \\
\left(S^{a}(V), \cdot, \Delta ?\right)
\end{gathered}
$$

free type

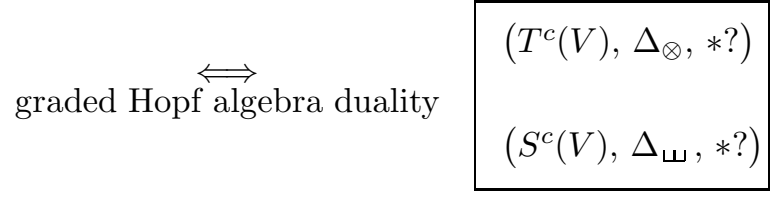

cofree type

Let us explain the diagram above: here, $V$ is a graded vector space with finite-dimensional homogeneous components, $T^{a}(V)$ stands for the free algebra on $V$ endowed with the tensor product $\otimes$ (also called concatenation on words), and $T^{c}(V)$ stands for the graded cofree coalgebra on $V$ endowed with the deconcatenation coproduct $\Delta_{\otimes}$ defined by:

$$
\Delta_{\otimes}\left(v_{1} \otimes \cdots \otimes v_{n}\right)=\sum_{k=0}^{n}\left(v_{1} \otimes \cdots \otimes v_{k}\right) \bigotimes\left(v_{k+1} \otimes \cdots \otimes v_{n}\right) .
$$

It can be seen as the graded dual of $T^{a}\left(V^{*}\right)$, where $V^{*}$ is the graded dual of $V$. In the same spirit, $S^{a}(V)$ stands for the free commutative algebra on $V$ endowed with the symmetrized tensor product ·, and $S^{c}(V)$ stands for the the graded cofree cocommutative coalgebra endowed with the unshuffle coproduct defined by:

$$
\Delta_{\boldsymbol{\omega}}(v)=v \otimes 1+1 \otimes v
$$

for any $v \in V$, and extended multiplicatively (see (6.9) below). The operations $\Delta$ ? and $*$ ? stand for a priori unknown coproducts and products which the give rise to Hopf algebras of the desired type.

J.-L. Loday and M. Ronco classified combinatorial Hopf algebras in [67]. The simplest examples are the coordinate rings of the pro-algebraic groups $G^{\text {inv }}$ and $G^{\text {dif }}$ and their graded duals, which are of the symmetric type, and their non-symmetric lifts. In particular, their results imply the existence of a brace bracket on the enveloping algebras of $L_{1}$ and $\mathcal{W}_{+}$, which reproduces the pre-Lie bracket already mentioned in section 5.2 .1 on the Lie algebras. We briefly discuss these examples.

6.2.1 The non-commutative Faà di Bruno Hopf algebra and the brace bracket The simplest examples of combinatorial Hopf algebras are the non-commutative lifts of the coordinate rings of the pro-algebraic groups $G^{\text {inv }}$ and $G^{\text {dif }}$, and their graded duals. In the case of the group $G^{\text {inv }}$, we obtain two combinatorial Hopf algebras:

$$
\begin{aligned}
& T^{a}(V)=\mathcal{H}_{\text {inv }}^{\text {nc }}=\mathbb{K}\left\langle x_{1}, x_{2}, x_{3}, \ldots\right\rangle, \quad \text { with product } \otimes \text { and coproduct } \Delta_{\text {inv }}, \\
& T^{c}(V)=\left(\mathcal{H}_{\text {inv }}^{\text {nc }}\right)^{*}=\mathbb{K}\left\langle x_{1}, x_{2}, x_{3}, \ldots\right\rangle, \quad \text { with coproduct }{ }^{t} \otimes=\Delta_{\otimes} \text { and product } *=\Delta_{\text {inv }}^{*} .
\end{aligned}
$$

We have already encountered the Hopf algebra $\mathcal{H}_{\text {inv }}^{\text {nc }}$ in section 4.5 : it is endowed with the same coproduct $\Delta_{\text {inv }}$ as $\mathcal{H}_{\text {inv }}$, and it is therefore cocommutative. Dually, the operation $*=\Delta_{\text {inv }}^{*}$ is the commutative product:

$$
x_{n} * x_{m}=x_{n+m},
$$

and $\left(\mathcal{H}_{\text {inv }}^{\text {nc }}\right)^{*}$ is then isomorphic to the tensor coalgebra on the polynomial $\operatorname{ring} \mathbb{K}[T]$ in one variable $T=x_{1}$, with isomorphism given by $x_{n} \mapsto T^{n}$. In the case of the group $G^{\text {dif }}$, we obtain two combinatorial Hopf algebras:

$$
\begin{aligned}
& T^{a}(V)=\mathcal{H}_{\mathrm{FdB}}^{\mathrm{nc}}=\mathbb{K}\left\langle x_{1}, x_{2}, x_{3}, \ldots\right\rangle, \quad \text { with product } \otimes \text { and coproduct } \Delta_{\mathrm{FdB}}, \\
& T^{c}(V)=\left(\mathcal{H}_{\mathrm{FdB}}^{\mathrm{nc}}\right)^{*}=\mathbb{K}\left\langle e_{1}, e_{2}, e_{3}, \ldots\right\rangle, \quad \text { with coproduct }{ }^{t} \otimes=\Delta_{\otimes} \text { and product } *=\Delta_{\mathrm{FdB}}^{*}
\end{aligned}
$$

\footnotetext{
${ }^{5}$ A different definition of combinatorial Hopf algebras was proposed, for commutative ones, by F. Hivert, J.-C. Novelli and J.-Y. Thibon in [51].
} 
The Hopf algebra $\mathcal{H}_{\mathrm{FdB}}^{\mathrm{nc}}$, and its mysterious nature, has already been discussed in sections 4.5 and 4.7 . For its dual Hopf algebra $\left(\mathcal{H}_{\mathrm{FdB}}^{\mathrm{nc}}\right)^{*}$, we shall refer to the general classification theorem established by Loday and Ronco: if $H \cong T^{c}(V)$ for some graded vector space $V$, with cofree coassociative coproduct $\Delta_{\otimes}$ and product $*$ satisfying the dual of the right-sided property (6.7), then $V$ is a (right) brace algebra [61], i.e. is equipped with a bilinear $\operatorname{map}\{;\}: V \otimes T(V) \longrightarrow V$, called (right) brace product, such that

$$
\left\{\left\{x ; y_{1} \cdots y_{p}\right\} ; z_{1} \cdots z_{q}\right\}=\sum\left\{x ; z_{1} \cdots z_{i_{1}}\left\{y_{1} ; z_{i_{1}+1} \cdots z_{j_{1}}\right\} z_{j_{1}+1} \cdots z_{i_{p}}\left\{y_{p} ; z_{i_{p}+1} \cdots z_{j_{p}}\right\} z_{j_{p}+1} \cdots z_{q}\right\}
$$

where the sum runs over all sequences $0 \leq i_{1} \leq j_{1} \leq \cdots \leq i_{p} \leq j_{p} \leq q$. In the case $T^{c}(V)=\left(\mathcal{H}_{\mathrm{FdB}}^{\mathrm{nc}}\right)^{*}$, the right-sided property is fulfilled by the opposite algebra $\left(\mathcal{H}_{\mathrm{FdB}}^{\mathrm{nc}, \mathrm{op}}\right)^{*}$, with product $\Delta_{\mathrm{FdB}}^{\mathrm{op}}{ }^{*}$ : in this case the brace product on the vector space $V=\operatorname{Span}_{\mathbb{K}}\left\{x_{1}, x_{2}, x_{3}, \ldots\right\}$ has been computed in [12]. If we omit the symbol $\otimes$ of the tensor product in the monomials, the brace product reads:

$$
\left\{x_{n} ; x_{m_{1}} \cdots x_{m_{q}}\right\}=\left(\begin{array}{c}
n+1 \\
q
\end{array}\right) x_{n+m_{1}+\cdots+m_{q}} .
$$

For the algebra $T^{c}(V)=\left(\mathcal{H}_{\mathrm{FdB}}^{\mathrm{nc}}\right)^{*}$, which satisfies the left-sided property, one gets a left brace product $\{;\}$ : $T(V) \otimes V \longrightarrow V$ satisfying the obvious analogue properties of (6.8).

6.2.2 The Faà di Bruno Hopf algebra and the pre-Lie bracket The symmetric versions of the two previous examples of combinatorial Hopf algebras are the coordinate rings of the pro-algebraic groups $G^{\text {inv }}$ and $G^{\text {dif }}$ (or $G^{\text {dif,op }}$ ), and their graded duals. In the case of the group $G^{\text {inv }}$, we get the two combinatorial Hopf algebras

$$
\begin{aligned}
& S^{a}(V)=\mathcal{H}_{\mathrm{inv}}=\mathbb{K}\left[x_{1}, x_{2}, x_{3}, \ldots\right], \quad \text { with free commutative product } \cdot \text { and coproduct } \Delta_{\mathrm{inv}}, \\
& S^{c}(V)=\mathcal{H}_{\mathrm{inv}}^{*}=\mathbb{K}\left\langle x_{1}, x_{2}, x_{3}, \ldots\right\rangle, \quad \text { with coproduct } \Delta_{\mathrm{w}} \text { and product } *=\Delta_{\mathrm{inv}}^{*} .
\end{aligned}
$$

The unshuffle coproduct is defined on a monomial $x_{n_{1}} \cdots x_{n_{k}} \in \mathbb{K}\left\langle x_{1}, x_{2}, x_{3}, \ldots\right\rangle$ (where we omit the tensor product $\otimes$ for simplicity) in the following way:

$$
\Delta_{\boldsymbol{\omega}}\left(x_{n_{1}} \cdots x_{n_{k}}\right)=\sum_{p+q=k} \sum_{\sigma \in S h(p, q)} x_{n_{\sigma(1)}} \cdots x_{n_{\sigma(p)}} \otimes x_{n_{\sigma(p+1)}} \cdots x_{n_{\sigma(p+q)}},
$$

where $\operatorname{Sh}(p, q)$ is the set of $(p, q)$-shuffles, i.e. the permutations $\sigma$ of $p+q$ numbers such that

$$
\sigma(1)<\cdots<\sigma(p) \quad \text { and } \quad \sigma(p+1)<\cdots<\sigma(p+q) .
$$

Both Hopf algebras are commutative and cocommutative: $\mathcal{H}_{\text {inv }}$ is the coordinate ring of the abelian group $G^{\text {inv }}(\mathbb{K})$, and $\mathcal{H}_{\text {inv }}^{*}$ is the enveloping algebra of the Lie algebra $\operatorname{Lie}\left(G^{\text {inv }}(\mathbb{K})\right)$, which is in fact the free abelian Lie algebra spanned by the variables $x_{1}, x_{2}, x_{3}, \ldots$, and is obviously isomorphic to the polynomial ring $K[T]$ in one variable.

In the case of the group $G^{\text {dif }}$, we get the two combinatorial Hopf algebras

$$
\begin{aligned}
& S^{a}(V)=\mathcal{H}_{\mathrm{FdB}}=\mathbb{K}\left[x_{1}, x_{2}, x_{3}, \ldots\right], \quad \text { with free commutative product } \cdot \text { and coproduct } \Delta_{\mathrm{FdB}}, \\
& S^{c}(V)=\mathcal{H}_{\mathrm{FdB}}^{*}=\mathbb{K}\left\langle e_{1}, e_{2}, e_{3}, \ldots\right\rangle, \quad \text { with coproduct } \Delta_{\boldsymbol{\omega}} \text { and product } *=\Delta_{\mathrm{FdB}}^{*} .
\end{aligned}
$$

In the symmetric case, the Loday-Ronco classification theorem (studied before also by T. Lada, M. Markl [61] and by D. Guin, J.-M. Oudom [49]) says the following: if $H \cong S^{c}(V)$ is endowed with the unshuffle coproduct $\Delta_{\boldsymbol{w}}$, and if the product $*$ satisfies the dual of the right-sided property (6.7), then $V$ is a right pre-Lie algebra, i.e. is equipped with a binary product $\triangleleft: V \otimes V \longrightarrow V$ such that:

$$
(x \triangleleft y) \triangleleft z-x \triangleleft(y \triangleleft z)=(x \triangleleft z) \triangleleft y-x \triangleleft(z \triangleleft y) .
$$

Comparing with the results in the non-symmetric case, a pre-Lie product is an example of a brace product $\{;\}: V \otimes T(V) \longrightarrow V$, which vanishes outside the subspace $V \otimes V$, that is

$$
\{x ; y\}=x \triangleleft y \quad \text { and } \quad\left\{x, y_{1} \cdots y_{m}\right\}=0 \quad \text { if } m \neq 1 .
$$

On the other side, any pre-Lie product on $V$ gives rise to a symmetric brace product $\{;\}: V \otimes S(V) \longrightarrow V$, 
recursively defined by

$$
\{x ; 1\}=x, \quad\{x ; y\}=x \triangleleft y, \quad \text { and } \quad\left\{x ; y_{1} \cdots y_{p}\right\}=\left\{x ; y_{1} \cdots y_{p-1}\right\} \triangleleft y_{p}-\left\{x ;\left\{y_{1} ; y_{2} \cdots y_{p-1}\right\}\right\} .
$$

Details can be found in [49] and [61].

This general result applies in particular to the Hopf algebra $\mathcal{H}_{\mathrm{FdB}}^{*}=U\left(L_{1}\right)$ (modulo a switch between right and left properties), and tells that the Lie algebra of vector fields $L_{1}$ is a symmetric left brace algebra, and thus a left pre-Lie algebra [12]: the opposite of the brace bracket given in (6.8) is in fact symmetric, and induces on $L_{1}$ the left pre-Lie product

$$
e_{n} \triangleleft e_{m}=\left\{e_{n} ; e_{m}\right\}^{o p}=(m+1) e_{n+m}
$$

that we saw in section 5.2.1.

Note that the pre-Lie bracket defined on $L_{1}$ can be extended to a left pre-Lie bracket on the Lie algebra $\operatorname{Vect}\left(\mathbb{S}^{1}\right)=\operatorname{Span}_{\mathbb{K}}\left\{e_{n}, n \in \mathbb{Z}\right\}$.

6.2.3 The shuffle Hopf algebra A last example of a combinatorial Hopf algebra is the shuffle Hopf algebra $T^{c}(V)=\mathbb{K}\left\langle x_{1}, x_{2}, \ldots\right\rangle$ endowed with the deconcatenation $\Delta_{\otimes}$ and the shuffle product

$$
\left(x_{n_{1}} \otimes \cdots \otimes x_{n_{p}}\right) \omega\left(x_{n_{p+1}} \otimes \cdots \otimes x_{n_{p+q}}\right)=\sum_{\sigma \in S h(p, q)} x_{n_{\sigma(1)}} \otimes \cdots \otimes x_{n_{\sigma(p+q)}} .
$$

We give the degree $n$ to the letter $x_{n}$. This commutative graded Hopf algebra represents the prounipotent group scheme $A \mapsto G_{A}$, whose Lie algebra is $\mathfrak{g}_{A}=\mathfrak{g} \otimes A$, where $\mathfrak{g}$ is the free Lie algebra over the alphabet $\left\{x_{1}, x_{2}, \ldots\right\}$.

\section{Operadic interpretation}

sect:operad Operads are combinatorial devices which appeared in algebraic topology, coined for coding "types of algebras". Hence, for example, a Lie algebra is an algebra over some operad denoted by Lie, an associative algebra is an algebra over some operad denoted by Assoc, a commutative algebra is an algebra over some operad denoted by Com, etc. The term "operad" has been invented by J.-P. May [73], although the notion itself appears a few years before in an article by J. M. Boardman and R. M. Vogt [8]. For a comprehensive and recent textbook on operads, we refer the reader to [68]. See also [64] for a more concise overview.

\subsection{Manipulating algebraic operations}

For any $n \in \mathbb{N}$, an $n$-ary operation on a vector space $V$ is an $n$-multilinear map

$$
a: V^{\otimes n} \longrightarrow V,\left(v_{1}, \ldots, v_{n}\right) \mapsto a\left(v_{1}, \ldots, v_{n}\right)
$$

suitably represented by a box:

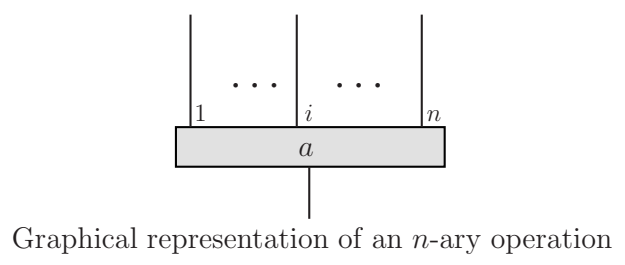

where the $n$ inputs represent the variables $v_{1}, \ldots, v_{n}$ and the output represents the result $a\left(v_{1}, \ldots, v_{n}\right)$ of the operation. For $n=1$ there is a canonical operation, namely the identity map Id $V \rightarrow V$. One can even consider 0-ary operations, as being just distinguished elements of $V$. 
Given an $n$-ary operation $a$, one can consider new $n$-ary operations by permuting the inputs of $a$, that is, by setting

$$
a^{\sigma}\left(v_{1}, \ldots, v_{n}\right)=a\left(v_{\sigma(1)}, \ldots, v_{\sigma(n)}\right)
$$

for any permutation $\sigma \in S_{n}$. For instance, given a binary operation $a\left(v_{1}, v_{2}\right)=v_{1} * v_{2}$, one can consider the opposite operation

$$
v_{1} *^{o p} v_{2}=v_{2} * v_{1}=a^{(12)}\left(v_{1}, v_{2}\right) .
$$

Given a ternary operation $b\left(v_{1}, v_{2}, v_{3}\right)$, one can define algtogether six operations $b_{\sigma}$ simply by permuting the inputs with a permutation $\sigma \in S_{3}$. In all cases, the symmetric group $S_{n}$ obviously acts (from the right) on the set of $n$-ary operations, by permuting their entries.

Given any two operations $a$ and $b$ on $V$, one also obtains new operations by applying them one after the other. For instance, if $a$ is an $n$-ary operation, and $b$ is an $m$-ary operation, we get an $(n+m-1)$-ary operation by applying first $b$ on any subset of $m$ variables among the $n+m-1$ available, and then applying $a$ to the $n$ variables thus obtained. The operation thus obtained is the partial composition $a \circ_{i} b$, for any choice of $i=1, \ldots, n$ where the result of $b$ is inserted as a variable of $a$, namely

$$
\left(a \circ_{i} b\right)\left(v_{1}, \ldots, v_{n+m-1}\right):=a\left(v_{1}, \cdots, v_{i-1}, b\left(v_{i}, \ldots, b_{i+m-1}\right), v_{i+m}, \ldots, v_{n+m-1}\right) .
$$

Graphically:

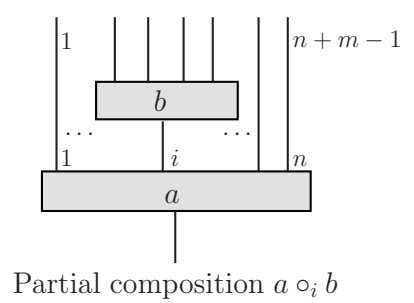

For instance, if we compose a binary operation $a\left(v_{1}, v_{2}\right)=v_{1} * v_{2}$ with itself, we obtain two ternary operations

$$
\left(v_{1}, v_{2}, v_{3}\right) \mapsto\left(v_{1} * v_{2}\right) * v_{3}, \quad \text { and } \quad\left(v_{1}, v_{2}, v_{3}\right) \mapsto v_{1} *\left(v_{2} * v_{3}\right)
$$

which do not change the order of the inputs, and twelve operations if we allow the permutations of the entries.

The "types of algebras" that one may wish to consider are characterized by the properties of the operations that one can perform. The concept of operad emerges when one tries to write such properties only in terms of the operations, discarding the entries. For example, a vector space $V$ is an associative algebra if it is endowed with a binary operation $a: V^{\otimes 2} \rightarrow V$, denoted by $*$, which is associative, i.e. for any $x, y, z \in V$ we have:

$$
(x * y) * z=x *(y * z) \quad \text { that is } \quad a \circ_{1} a=a \circ_{2} a .
$$

Similarly, a vector space $V$ is a Lie algebra if it is endowed with a binary operation $a: V^{\otimes 2} \rightarrow V$, denoted by $[$,$] , which is antisymmetric and satisfies the Jacobi identity, i.e. for any x, y, z \in V$ we have:

$$
\begin{aligned}
{[x, y]+[y, x] } & =0 \text { that is } a+a^{(12)}=0, \\
{[[x, y], z]+[[y, z], x]+[[z, x], y] } & =0 \text { that is } a \circ_{1} a+\left(a \circ_{1} a\right)^{\tau}+\left(a \circ_{1} a\right)^{\tau^{2}}=0,
\end{aligned}
$$

where $\tau$ is the circular permutation $(123) \in S_{3}$.

\subsection{Algebraic operads}

An algebraic operad, or linear operad, is a collection $\mathcal{P}=(\mathcal{P}(n))_{n \geq 0}$ of $\mathbb{K}$-vector spaces $\mathcal{P}(n)$ on which the symmetric group $S_{n}$ acts from the right, together with a distinguished element $e \in \mathcal{P}(1)$, called the identity, and a collection of linear maps, called partial compositions,

$$
\begin{aligned}
\circ_{i}: \mathcal{P}(k) \otimes \mathcal{P}(l) & \longrightarrow \mathcal{P}(k+l-1), \quad i=1, \ldots, k \\
(a, b) & \longmapsto a \circ_{i} b
\end{aligned}
$$

satisfying the following associativity, unit and equivariance axioms: 
- The nested and the disjoint associativity: for any $a \in \mathcal{P}(k), b \in \mathcal{P}(l), c \in \mathcal{P}(m)$ one has:

$$
\begin{array}{ll}
\left(a \circ_{i} b\right) \circ_{i+j-1} c=a \circ_{i}\left(b \circ_{j} c\right), & i \in\{1, \ldots, k\}, \quad j \in\{1, \ldots, l\}, \\
\left(a \circ_{i} b\right) \circ_{l+j-1} c=\left(a \circ_{j} c\right) \circ_{i} b, & i, j \in\{1, \ldots, k\}, \quad i<j .
\end{array}
$$

Graphically, these compositions produce the following operations:
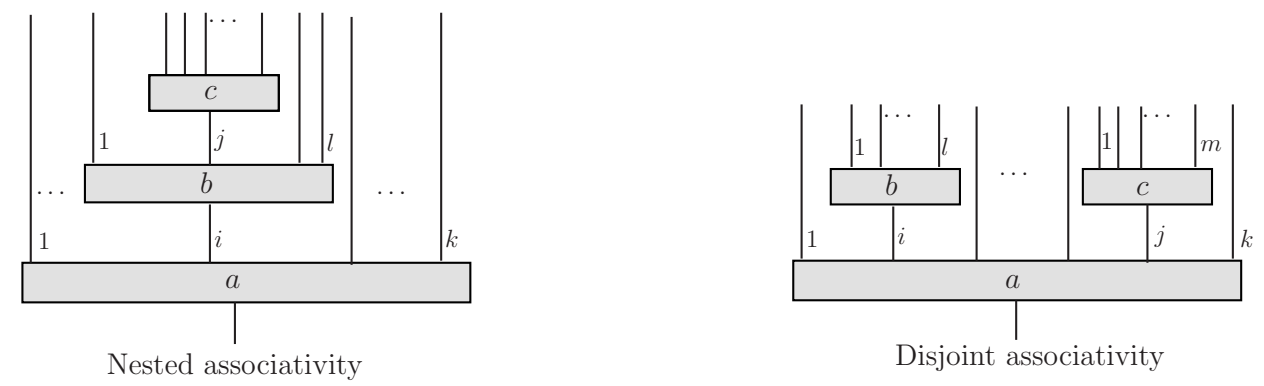

- The unit property:

$$
\begin{aligned}
e \circ a & =a \\
a \circ_{i} e & =a, \quad i=1, \ldots, k .
\end{aligned}
$$

- The equivariance property:

$$
a^{\sigma} \circ_{\sigma(i)} b^{\tau}=\left(a \circ_{i} b\right)^{\eta_{i}(\sigma, \tau)}
$$

where $\eta_{i}(\sigma, \tau) \in S_{k+l-1}$ is defined by letting $\tau$ permute the set $E_{i}=\{i, i+1, \ldots, i+l-1\}$ of cardinality $l$, and then by letting $\sigma$ permute the set $\left\{1, \ldots, i-1, E_{i}, i+l, \ldots, k+l-1\right\}$ of cardinality $k$.

The prototype of algebraic operads is the endomorphism operad on a vector space $V$, denoted by $\operatorname{End}(V)$. For any $n \geq 0$, it is given by the vector space of $n$-multilinear maps on $V$, that is,

$$
\operatorname{End}(V)(n)=\mathcal{L}\left(V^{\otimes n}, V\right) .
$$

The right action of the symmetric group $S_{n}$ on $\operatorname{End}(V)(n)$ is induced by the left action of $S_{n}$ on $V^{\otimes n}$ given by:

$$
\sigma \cdot\left(v_{1} \otimes \cdots \otimes v_{n}\right)=v_{\sigma_{1}^{-1}} \otimes \cdots \otimes v_{\sigma_{n}^{-1}}
$$

The unit element is the identity map $e: V \rightarrow V$, and the partial compositions are given by true compositions of linear maps.

Given an operad $\mathcal{P}$, a $\mathcal{P}$-algebra is a vector space $A$ together with a morphism of operads from $\mathcal{P}$ to $\operatorname{End}(A)$, that is, for any $n \geq 0$, an equivariant linear map

$$
\mathcal{P}(n) \longrightarrow \operatorname{End}(A)(n)=\mathcal{L}\left(A^{\otimes n}, A\right)
$$

which identifies each element $a$ of $\mathcal{P}(n)$ with an $n$-ary operation on $A$, and which preserves the partial compositions. Given two $\mathcal{P}$-algebras $A$ and $B$, a morphism of $\mathcal{P}$-algebras from $A$ to $B$ is a linear map $f: A \rightarrow B$ such that, for any $n \geq 0$ and for any $a \in \mathcal{P}(n)$, the following diagram commutes,

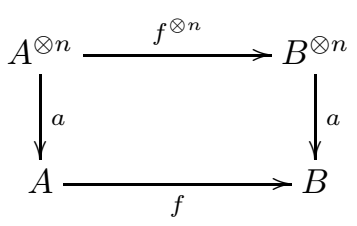

where we have denoted by the same letter $a$ the element of $\mathcal{P}(n)$ and its images in $\operatorname{End}(A)(n)$ and $\operatorname{End}(B)(n)$. 
Using the partial compositions one can also define a total composition

$$
\begin{aligned}
\gamma: \mathcal{P}(n) \otimes \mathcal{P}\left(k_{1}\right) \otimes \cdots \otimes \mathcal{P}\left(k_{n}\right) & \longrightarrow \mathcal{P}\left(k_{1}+\cdots+k_{n}\right) \\
\left(a, b_{1}, \ldots, b_{n}\right) & \longmapsto \gamma\left(a ; b_{1}, \ldots, b_{n}\right)=\left(\ldots\left(\left(a \circ_{n} b_{n}\right) \circ_{n-1} b_{n-1}\right) \cdots\right) \circ_{1} b_{1},
\end{aligned}
$$

which is graphically represented as follows:

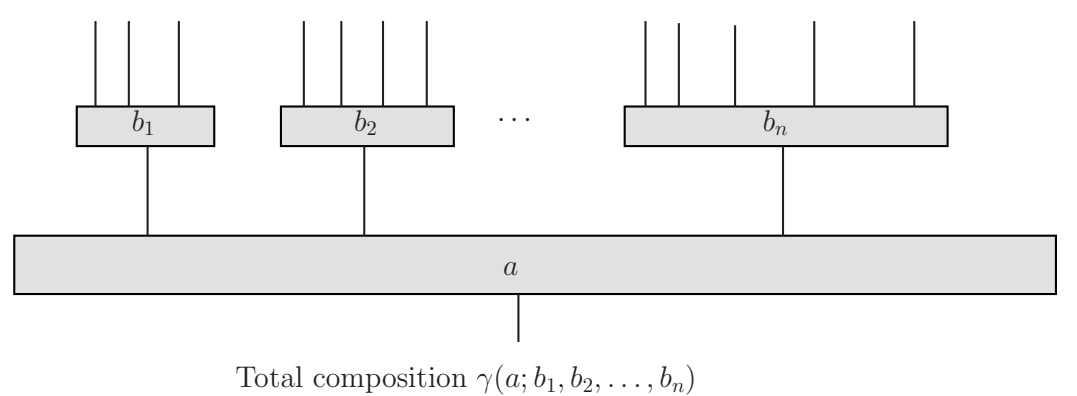

Such a map allows us to regard an operad $\mathcal{P}$ as a Schur functor [69, 68] on $\mathbb{K}$-vector spaces:

$$
\mathcal{P}: \operatorname{Vect}_{\mathbb{K}} \longrightarrow \operatorname{Vect}_{\mathbb{K}}, V \longmapsto \mathcal{P}(V)=\bigoplus_{n \geq 0} \mathcal{P}(n) \otimes_{S_{n}} V^{\otimes n}
$$

endowed with an associative composition $\gamma: \mathcal{P} \circ \mathcal{P} \longrightarrow \mathcal{P}$ and with a unit $i: \mathcal{I} \hookrightarrow \mathcal{P}$ from the trivial operad $\mathcal{I}(n)=\delta_{n, 1} \mathbb{K} e$. A $\mathcal{P}$-algebra can then be also seen as a vector space $A$ endowed with an associative linear map $\gamma_{A}: \mathcal{P} \circ \mathcal{P}(A) \longrightarrow \mathcal{P}(A)$, i.e. such that $\gamma_{A}\left(\gamma_{A} \circ I\right)=\gamma_{A}\left(I \circ \gamma_{A}\right): \mathcal{P} \circ \mathcal{P} \circ \mathcal{P}(A) \longrightarrow \mathcal{P}(A)$. The usual type of algebras give rise to the following operads:

- Assoc for associative algebras: $A \operatorname{ssoc}(n)=\mathbb{K}\left[S_{n}\right]$.

- $C o m$ for associative and commutative algebras: $\operatorname{Com}(n)$ is given by the trivial representation of $S_{n}$.

- Lie for Lie algebras: the vector space $\operatorname{Lie}(n)$ is the representation of $S_{n}$ induced from the one-dimensional representation $\rho$ of $C_{n}=\mathbb{Z} / \mathbb{Z}_{n}$ given by a primitive $n$-th root of unity, i.e. $\operatorname{Lie}(n)=\operatorname{Ind}_{C_{n}}^{S_{n}}(\rho)$.

- preLie for right pre-Lie algebras $[42,84]$ : as a vector space $\operatorname{preLie}(n)=\operatorname{Span}_{\mathbb{K}}\left\{\mathcal{T}_{n}\right\}$ is $\operatorname{spanned}$ by the rooted trees with $n$ labeled vertices, and the symmetric groups $S_{n}$ acts by permuting the labels [19].

For more details about operads, see e.g. [64, 68].

\subsection{Pre-Lie algebras, Lie algebras and groups associated to operads}

For operads $\mathcal{P}$ such that $\mathcal{P}(0)=\{0\}$, there is a canonical way to construct a pronilpotent Lie algebra and, dually, a prounipotent group. For simplicity, let us illustrate this construction for regular operads.

An operad $\mathcal{P}$ is regular [68, Paragraph 5.2.9] if for any $n \geq 0$ we have $\mathcal{P}(n)=\mathcal{P}_{n} \otimes \mathbb{K}\left[S_{n}\right]$, where $\mathcal{P}_{n}$ is some vector space. In this case, the operad $\mathcal{P}$ gives rise to the non-symmetric operad $\mathcal{P}^{\prime}=\left(\mathcal{P}_{n}\right)_{n \geq 0}$, which verifies axioms similar to those of an operad, except that the actions of symmetric groups are not required. Any operad is also a non-symmetric operad, if we forget the symmetric group actions. An algebra over a non-symmetric operad $\mathcal{P}^{\prime}$ is a vector space $A$ together with a non-symmetric operad morphism from $\mathcal{P}^{\prime}$ into the underlying non-symmetric operad of $\operatorname{End}(A)$.

An operad $\mathcal{P}$ is regular if the relations defining the $\mathcal{P}$-algebras can be written without switching the arguments. For example, the associativity identity $(x * y) * z=x *(y * z)$ does not change the order of the variables: thus $A s s o c$ is regular. Indeed $A \operatorname{ssoc}(n)=\mathbb{K}\left[S_{n}\right]$, and the associated non-symmetric operad $A s s o c^{\prime}$ is given by $A s \operatorname{soc}_{n}^{\prime}=\mathbb{K}$. The operads Com, Lie and preLie are not regular. 
Let $\mathcal{P}$ be a regular operad, hence $\mathcal{P}(n)=\mathcal{P}_{n} \otimes \mathbb{K}\left[S_{n}\right]$. The Lie algebra associated to $\mathcal{P}$ was introduced by M. Kapranov in 2000, see [57], as the vector space

$$
L^{\mathcal{P}}=\bigoplus_{n=2}^{\infty} \mathcal{P}_{n}=\left\{\sum_{\text {finite }} p_{n} \mid p_{n} \in \mathcal{P}_{n}\right\}
$$

with Lie bracket $[p, q]=p \triangleleft q-q \triangleleft p$ induced by the right pre-Lie product

$$
p \triangleleft q=\sum \gamma(q ; \mathrm{id}, \ldots, p, \ldots, \mathrm{id}),
$$

where the sum runs on the possible places to put $p$. Since $\mathcal{P}_{n} \triangleleft \mathcal{P}_{m} \subset \mathcal{P}_{n+m-1}$, the above pre-Lie and Lie products are graded by $|p|=n-1$ for $p \in \mathcal{P}_{n}$. As the sum starts at $n=2$, this Lie algebra is pronilpotent. The completion $\widehat{L^{\mathcal{P}}}$ of the Lie algebra $L^{\mathcal{P}}$, with respect to this graduation, contains formal series, i.e. possibly infinite sums $\sum_{n \geq 2} p_{n}$, with $p_{n} \in \mathcal{P}_{n}$.

If the operad $\mathcal{P}$ is not regular, a similar construction makes sense if we consider the sum of coinvariant spaces, with respect to the action of the symmetric group, that is, if we set:

$$
L^{\mathcal{P}}=\bigoplus_{n=2}^{\infty} \mathcal{P}(n) / S_{n}
$$

Pre-Lie and Lie products are defined similarly, as the sum of all partial compositions still makes sense modulo the symmetric group actions.

Let $\mathcal{P}$ be a regular operad which satisfies $\mathcal{P}(0)=\{0\}$ and $\mathcal{P}(1)=\mathbb{K} e$. The group associated to $\mathcal{P}$ was defined independently by F. Chapoton [18] and P. van der Laan [82] as the set

$$
G^{\mathcal{P}}=\left\{\sum_{n=1}^{\infty} p_{n} \mid p_{n} \in \mathcal{P}_{n} \text { and } p_{1}=e\right\}
$$

with the composition law:

$$
\sum_{n \geq 1} p_{n} \circ \sum_{m \geq 1} q_{m}=\sum_{n \geq 1} \sum_{m_{1}, \ldots m_{n} \geq 1} \gamma\left(p_{n} ; q_{m_{1}}, \ldots, q_{m_{n}}\right) .
$$

A similar construction is again possible for non-regular operads, if we replace $\mathcal{P}_{n}$ by $\mathcal{P}(n) / S_{n}$. The group $G^{(\mathcal{P})}$ is prounipotent, with associated pronilpotent Lie algebra $\widehat{L^{\mathcal{P}}}$.

Two Hopf algebras can then be associated to $\mathcal{P}$ : the co-commutative Hopf algebra $U\left(L^{\mathcal{P}}\right)$, and its graded dual $H^{\mathcal{P}}$, which is commutative and represents the proalgebraic group $G^{\mathcal{P}}$, i.e. $G^{\mathcal{P}}(A)=\operatorname{Hom} \mathrm{CAlg}\left(H^{\mathcal{P}}, A\right)$ for any unital and commutative algebra $A$.

7.3.1 The Faà di Bruno Hopf algebra and the associative operad F. Chapoton and P. van der Laan proved $[18,82]$ that the group $G^{\mathcal{P}}$ is the group of formal diffeomorphisms $G^{\text {dif }}$ if $\mathcal{P}$ is the non-symmetric operad Assoc of associative algebras. In this case, in fact, in each degree $n \geq 1$ there is only one possible operation, up to a scalar factor and permutation. If we choose a generator $p_{n}$ for each vector space $A s s o c_{n}^{\prime}$ (with the notation introduced in the beginning of Section 7.3), an element of the group $G^{\mathcal{P}}$ is a formal series

$$
f=\sum_{n=1}^{\infty} f_{n} p_{n}, \quad \text { with } f_{n} \in \mathbb{K} \text { and } f_{1}=1,
$$

and the operadic composition of two such series gives exactly the Faà di Bruno composition of diffeomorphisms. If $\mathcal{P}$ is the (non-regular) operad Com of commutative algebras, the construction above gives back the group $G^{\text {inv }}[18]$.

A similar construction of the group $G^{\text {dif }}$ was done in [37] for set-operads. In this case, there was proven a general criterion to compare the group $G^{\text {dif }}$ with the group $G^{\mathcal{P}}$ associated to suitable operads. An operad $\mathcal{P}$ is a set-like operad if the relations defining the operations do not involve sums or multiplications by scalars. For instance, the operads Assoc and Com are set-like. A set-like operad $\mathcal{P}$ is then the collection of the vector 
spaces $\mathcal{P}(n)$ generated by some sets $\mathcal{P}_{\text {set }}(n)$. The collection $\mathcal{P}_{\text {set }}=\left(\mathcal{P}_{\text {set }}(n)\right)_{n \geq 1}$ gives rise to a set operad, that is, an operad in the category of sets. The axioms for set operads are the same as for linear operads, except that the tensor product of vector spaces is replaced by the cartesian product of sets, and the direct sum is replaced by the disjoint union. If moreover the set-like operad is regular, the associated set operad is regular as well, i.e. $\mathcal{P}_{\text {set }}(n)=\mathcal{P}_{\text {set }, n} \times S_{n}$ for any $n \geq 1$.

If $\mathcal{P}$ is a regular set-like operad, the proalgebraic group $G^{\mathcal{P}}$ was called the group of $\mathcal{P}$-expanded series in [37]:

$$
G^{\mathcal{P}}(A)=\left\{f(x)=\sum_{p \in \mathcal{P}_{n}} f_{p} x^{p} \mid f_{p} \in A\right\}
$$

where $x^{p}$ is just a symbol, with product given by a formal composition law

$$
(f \circ g)(x)=\sum_{p \in \mathcal{P}} \sum_{q_{1}, \ldots, q_{|p|} \in \mathcal{P}} f_{p} g_{q_{1}} \cdots g_{q_{|p|}} x^{\gamma\left(p, q_{1}, \ldots, q_{|p|}\right)} .
$$

The simplest example of a regular set-like operad is Assoc, with one single $n$-ary operation up to permutation for any $n \geq 1$ which is denoted by $n-1$. Hence the non-symmetric set operad $A s s o c_{\text {set }}^{\prime}$ can be identified with the set $\mathbb{N}$ of (non-negative) natural numbers. The operadic partial composition of two operations $p$ and $q$ (which is unique by virtue of the associativity) coincides with the sum $p+q$. Similarly, the total composition $\gamma\left(n ; m_{0}, m_{1}, \ldots, m_{n}\right)$ coincides with the sum $m_{0}+\cdots+m_{n}$. For any unital commutative algebra $A$, the group $G^{A s s o c_{\text {set }}^{\prime}}(A)$ is again the group of formal diffeomorphisms $G^{\text {dif }}(A)$. In [37] it was shown also that, for any regular set operad $\mathcal{P}$, the order map

$$
\mathcal{P} \longrightarrow A s \operatorname{soc}_{\mathrm{set}}^{\prime}, \quad p \mapsto|p|
$$

induces a canonical surjective morphism of groups $G^{\mathcal{P}}(A) \rightarrow G^{\mathrm{dif}}(A)$. Moreover, any element $p_{2} \in \mathcal{P}_{2}$ which satisfies the associativity axiom, if it exists, gives an operad morphism

$$
\operatorname{Assoc}_{\mathrm{set}}^{\prime} \longrightarrow \mathcal{P}, \quad n \longmapsto p_{n}=\gamma\left(p_{2} ; p_{n-1}, e\right)
$$

which induces a section $G^{\operatorname{dif}}(A) \longrightarrow G^{\mathcal{P}}(A)$.

7.3.2 The QED charge Hopf algebra on planar binary trees and the duplicial operad Another example of a regular set operad is the operad Dup which defines duplicial algebras [89], that is, algebras $A$ endowed with two binary operations over / and under \, satisfying the following identities:

$$
\begin{aligned}
& (x / y) / z=x /(y / z) \\
& (x / y) \backslash z=x /(y \backslash z) \\
& (x \backslash y) \backslash z=x \backslash(y \backslash z),
\end{aligned}
$$

for any $x, y, z \in A$. The operad Dup is described by means of planar binary trees [37, 65], that is, planar rooted trees with internal vertices of valence 3 . For any $n \geq 0, D u p_{n}$ is the set of trees with $n$ internal vertices:

$$
\begin{aligned}
& \operatorname{Dup}_{0}=\{\mid\}, \quad \operatorname{Dup}_{1}=\{Y\}, \quad \operatorname{Dup}_{2}=\{Y, Y\}, \\
& \operatorname{Dup}_{3}=\{Y, Y, Y, Y, Y, Y\} .
\end{aligned}
$$

The operations over and under are defined on any two trees $t$ and $s$ as the following grafting of the root of a tree onto the left-most or onto the right-most leaf of the other one:

$$
\begin{aligned}
t \text { over } s: & t / s={ }^{t} s \\
t \text { under } s: & t \backslash s=t^{\prime}
\end{aligned}
$$

The operadic composition in Dup is then given by the following rule: for any trees $t$ and $s_{1}, \ldots, s_{|t|}$, the tree $\gamma\left(t ; s_{1}, \ldots, s_{|t|}\right)$ is obtained by replacing each internal vertex of $t$ by the trees $s_{1}, \ldots, s_{|t|}$, in the order given by 
the decomposition of $t$ as a monomial in the vertex tree $Y$, using the over and under products and suitable parentheses. For instance,

$$
Y=(Y \backslash Y) / Y \text { hence } \mu_{Y}\left(s_{1}, s_{2}, s_{3}\right)=\left(s_{1} \backslash s_{2}\right) / s_{3}={ }^{s_{1}^{\prime}} s_{3} .
$$

The group $G^{D u p}(A)$ of tree-expanded series of the form

$$
f(x)=\sum_{t \in \text { Dup }} f_{t} x^{t}, \quad \text { with } f_{t} \in A \text { and } f_{Y}=1
$$

was studied extensively in [37]. Since the operad Dup contains a binary associative operation Y, the group $G^{D u p}(A)$ projects onto the group $G^{\text {dif }}(A)$ and at the same time contains a copy of $G^{\text {dif }}(A)$. Moreover, this proalgebraic group is represented by a commutative Hopf algebra $H_{D u p}$ on planar binary trees which admits a non-commutative lift different from the Loday-Ronco Hopf algebra on planar binary trees [66], and different from its linear dual.

Finally, the group $G^{D u p}(\mathbb{C})$ contains a proalgebraic subgroup $G^{\alpha}(\mathbb{C})$ whose representative Hopf algebra $H^{\alpha}$ was used in $[10,11]$ to describe the renormalization of the electric charge in massless quantum electrodynamics. The algebra $H^{\alpha}$ is a suitable quotient of the algebra $H_{D u p}$, such that the group $G^{\alpha}(\mathbb{C})$ can be seen as the set of tree-expanded series of the form

$$
\alpha_{f}(x)=\left(x^{\mid}-x^{Y} \backslash f(x)\right)^{-1} / x Y
$$

for any tree-expanded series $f(x)=\sum f_{t} x^{t}$. Here the inversion of the series $x \mid-x Y \backslash f(x)$ is performed in the group of tree-expanded invertible series, which is the set of tree-expanded series starting with the term $x \mid$, and considered with the product over.

\section{References}

[1] L. F. A. Arbogast, Du calcul des dérivations, Levrault, Strasbourg (1800).

[2] S. Agarwala, The Geometry of Renormalization, Ph.D. Thesis, Johns Hopkins University (2008).

[3] E. T. Bell, Exponential polynomials, Ann. of Math. (2) 35 (1934), 258-277.

[4] M. Bellon, F. Schaposnik, Renormalization group functions for the Wess-Zumino model: up to 200 loops through Hopf algebras, Nucl. Phys. A800 (2008), 517-526.

[5] G. M. Bergman, A. O. Hausknecht, Co-groups and co-rings in categories of associative rings, Math. Surveys and Monographs 45, Amer. Math. Soc. (1996).

[6] I. Berstein, On co-groups in the category of graded algebras, Trans. Amer. Math. Soc. 115 (1965), 257-269.

[7] R. E. Block, On the Mills-Seligman axioms for Lie algebras of classical type, Trans. Amer. Math. Soc., 121 (1966), 378-392

[8] J. M. Boardman, R. M. Vogt, Homotopy-everything H-spaces, Bull. Amer. Math. Soc. 74 No 6 (1968), $1117-1122$.

[9] C. Brouder, On the trees of quantum fields, Eur. Phys. J. C 12 (2000), 535-549.

[10] C. Brouder and A. Frabetti, Renormalization of QED with planar binary trees, Eur. Phys. J. C 19 (2001), 715-741.

[11] C. Brouder and A. Frabetti, QED Hopf algebras on planar binary trees, J. Alg. 267 (2003), 298-322.

[12] C. Brouder, A. Frabetti, F. Menous, Combinatorial Hopf algebras from renormalization, J. Alg. Combinatorics 32 (2010), 557-578.

[13] J. Butcher, Coefficients for the study of Runge-Kutta integration processes, J. Austral. Math. Soc. 3 No2, 185-201 (1963).

[14] J. Butcher, An algebraic theory of integration methods, Math. Comp. 26 (1972), 79-106.

[15] C. Brouder, A. Frabetti and C. Krattenthaler, Non-commutative Hopf algebra of formal diffeomorphisms, Adv. Math. 200 (2006), 479-524. 
[16] E. Cartan, Les groupes de transformations continus, infinis, simples, Ann. Sci. Ecole Norm. Sup. 26 (3) (1909), 93-161.

[17] P. Cartier, A primer of Hopf algebras, Frontiers in Number Theory, Physics and Geometry II, 537-615, Springer (2007).

[18] F. Chapoton, Rooted trees and exponential-likes series, preprint, arXiv:math/0209104

[19] F. Chapoton, M. Livernet, Pre-Lie algebras and the rooted trees operad, Int. Math. Res. Notices 8 (2001), 395-408.

[20] A. Connes and D. Kreimer, Hopf Algebras, Renormalization and Noncommutative Geometry. Comm. Math. Phys. 199 (1998) 203-242.

[21] A. Connes and D. Kreimer, Renormalization in quantum field theory and the Riemann-Hilbert problem. I. The Hopf algebra structure of graphs and the main theorem. Comm. Math. Phys. 210 (2000), 249-273.

[22] A. Connes and D. Kreimer, Renormalization in quantum field theory and the Riemann-Hilbert problem. II. The $\beta$-function, diffeomorphisms and the renormalization group. Comm. Math. Phys. 216 (2001), 215-241.

[23] A. Connes, H. Moscovici, Hopf algebras, cyclic cohomology and the transverse index theorem, Comm. Math. Phys. 198 (1998), 199-248.

[24] A. D. D. Craik, Prehistory of Faà di Bruno's formula, Amer. Math. Monthly 112 No2 (2005), 119-130.

[25] P. Doubilet, A Hopf algebra arising from the lattice of partitions of a set, J. Algebra 28 (1986), 127-132.

[26] A. Dür, Möbius functions, incidence algebras and power series representations, Lect. Notes math. 1202, Springer (1986).

[27] K. Ebrahimi-Fard and L. Guo, Rota-Baxter Algebras in Renormalization of Perturbative Quantum Field Theory, Fields Institute Communications 50 (2007), 47-105.

[28] K. Ebrahimi-Fard, F. Patras, Exponential renormalization, Ann. Henri Poincaré 11 (5), (2010), 943-971.

[29] K. Ebrahimi-Fard, F. Patras, Exponential Renormalization II: Bogoliubov's R-operation and momentum subtraction schemes, J. Math. Phys. 53, 083505 (2012). arXiv:1104.3415v1 (2011).

[30] B. Eckmann, P. J. Hilton, Group-like structures in general categories I, II and III, Math. Ann. 145 (1962), 227-255, ibid. 151 (1963), 150-186 and ibid. 150 (1963), 165-187.

[31] R. Ehrenborg, On posets and Hopf algebras, Adv. Math. 119 (1996), 1-25.

[32] F. Faà di Bruno, Sullo sviluppo delle funzioni, Ann. Sci. Mat. Fis., Roma 6 (1855), 479-480.

[33] H. Figueroa, J. Gracia-Bondia, J. Varilly, Faà di Bruno Hopf algebras, arXiv:math/0508337 (2005).

[34] H. Figueroa, J. Gracia-Bondia, Combinatorial Hopf algebras in quantum field theory I, Rev. Math. Phys. 17 (2005), $881-976$.

[35] L. Foissy, Les algèbres de Hopf des arbres enracinés décorés I,II, Bull. Sci. Math. 126 (2002), 193-239 and 249-288.

[36] L. Foissy, Faà di Bruno subalgebras of the Hopf algebra of planar trees from combinatorial Dyson-Schwinger equations, Adv. Math. 218 (2008), 136-162.

[37] A. Frabetti, Groups of tree-expanded formal series, Journal of Algebra 319 (2008), 377-413.

[38] I. Frenkel, J. Lepowsky, A. Meurmann, Vertex operator algebras and the Monster Pure and Appl. Math. 134, Academic Press, New York (1988).

[39] R. Frucht, G-C. Rota, Polinomios de Bell y particiones de conjuntos finitos, Scientia 126 (1965), 5-10.

[40] D. B. Fuks, Cohomology of infinite-dimensional Lie algebras, Consultants Bureau, New York 1986.

[41] D. B. Fuks, I. M. Gelfand, Cohomology of Lie algebras of vector fields on the circle, Funkts. Anal. Prilozhen. 2 No. 4 (1968), 92-93.

[42] M. Gerstenhaber The cohomology structure of an ass-ociative ring, Ann. Math. 78, No2 (1963), 267-288.

[43] I. M. Gelfand, D. Krob, A. Lascoux, B. Leclerc, V. Retakh and J.-Y. Thibon, "Noncommutative symmetric functions", Adv. Math. 112 (1995), 218-348.

[44] L. Giacardi, Francesco Faà di Bruno. Ricerca scientifica, insegnamento e divulgazione, Deputazione subalpina di storia patria, Studi e fonti XII, Torino (2004).

[45] F. Girelli, T. Krajewski, P. Martinetti, An algebraic Birkhoff decomposition for the continuous renormalization group, J. Math. Phys. 45 (2004), 4679-4697.

[46] P. Goddard, D. Olive eds., Kac-Moody and Virasoro algebras, a reprint volume for physicists, Adv. Series in Math. Phys. 3, World Scientific (1988). 
[47] R. Grossman, R. G. Larson, Hopf-algebraic structure of families of trees, J. Algebra 126, No 1 (1989), $184-210$.

[48] W. S. Gray, L. A. Duffaut Espinosa, A Faà di Bruno Hopf algebra for a group of Fliess operators with applications to feedback, Systems \& control Lett. 60 (7) (2011), 441-449.

[49] D. Guin, J.-M. Oudom, On the Lie enveloping algebra of a pre-Lie algebra, J. of K-theory: K-theory and its applications to algebra, geometry and topology 2 issue 01 (2008) 147-167.

[50] E. Hairer, Ch. Lubich, G. Wanner, Geometric numerical integration: structure-preserving algorithms for ordinary differential equations, 2nd edition, Springer (2006).

[51] F. Hivert, J.-C. Novelli, J.-Y. Thibon, Commutative combinatorial Hopf algebras, J. Alg. Combinatorics 28 No 1 (2008), 65-95.

[52] G. Hochschild, La structure des groupes de Lie, Dunod 1968.

[53] W. P. Johnson, The curious history of Faà di Bruno's formula, AMS Monthly 109 (2002), 217-234.

[54] S. A. Joni, G.-C. Rota, Coalgebras and bialgebras in combinatorics, Stud. Appl. Math. 61 No2 (1979), $145-163$.

[55] V. Kac, A. Raina, Bombay lectures on highest weight representations of infinite-dimensional Lie algebras, Adv. Series in Math. Phys. 2, World Scientific (1987).

[56] V. Kac, Vertex algebras for beginners, Univ. Lectures Series 10, Amer. Math. Soc., Providence (1997).

[57] M. Kapranov, Yu. Manin, Modules and Morita theorem for operads, Amer. J. Math. 125, No5 (2001), 811-838.

[58] T. Knight, On the expansion of any functions of multinomials, Philos. Trans. R. Soc. London 101 (1811), $49-88$.

[59] D. Kreimer, On the Hopf algebra structure of perturbative quantum field theories, Adv. Theor. Math. Phys.2 (1998), 303-334.

[60] S. F. Lacroix, Traité du calcul différentiel et du calcul intégral, (3 volumes), Duprat, Paris, 1810-1819.

[61] T. Lada, M. Markl, Symmetric brace algebras, Appl. Categorical Structures 13, Issue 4 (2005), 351-370.

[62] J.-L. Lagrange, Nouvelle méthode pour résoudre les équations littérales par le moyen des séries, Mémoires de l'Académie Royale des Sciences et Belles-Lettres de Berlin 24 (1770) 251-326.

[63] J. Lepowsky, H. Li, Introduction to vertex operator algebras and their representations, Progress in Math. 227, Birkhäuser, Boston (2003).

[64] J-L. Loday, La renaissance des opérades, Séminaire Bourbaki, Vol. 1994/95. Astérisque 237, Exp. No. 792, 3 (1996), 47-74.

[65] J.-L. Loday, Generalized bialgebras and triples of operads, Astérisque 320 (2008), 116 pages.

[66] J.-L. Loday, M. Ronco, Hopf algebra of the planar binary trees, Adv. Math. 139 (1998), 293-309.

[67] J.-L. Loday, M. Ronco, Combinatorial Hopf algebras, Quanta of Math., Clay Math. Proc. 10 (2008), $347-383$.

[68] J-L. Loday, B. Vallette, Algebraic operads, Grund. Math. Wiss. 346, Springer (2012). www-irma.ustrasbg.fr/ loday/

[69] I. G. Macdonald, Symmetric functions and Hall polynomials, Oxford University Press 1979.

[70] S. Mac Lane, Categories for the working mathematician, Second edition, Springer (1998).

[71] D. Manchon Hopf algebras and renormalisation, Handbook of algebra 5 (M. Hazewinkel ed.) (2008), 365-427.

[72] D. Manchon, On bialgebras and Hopf algebras of oriented graphs, Confluentes Math. 4 No1 (2012), 10 pages, arXiv: 1011:3032.

[73] J. P. May, The Geometry of Iterated Loop Spaces, Springer-Verlag (1972).

[74] F. Menous, Formulas for the Connes-Moscovici Hopf algebra, C. R. Math. Acad. Sci. Paris 341 (2) (2005), 75-78.

[75] R. Ree, Lie elements and the algebra associated with shuffles, Ann. Math. 68 No2 (1958), 210-219.

[76] J. Riordan, Derivatives of composite functions, Bull. Amer. Math. Soc. 52 (1946), 664-667.

[77] G.-C. Rota, On the foundations of combinatorial theory I: theory of Möbius functions, Z. Wahrscheinlichkeitstheorie and Werw. Gebiete 2 (1964), 340-368.

[78] W. R. Schmitt, Incidence Hopf algebras, Journal of Pure and Applied Algebra 96 (1994), 299-330.

[79] R. P. Stanley, Enumerative combinatorics, 2nd. edition, Cambridge University Press (1997).

[80] M. E. Sweedler, Hopf algebras, Benjamin (1969).

[81] E. C. Titchmarsh, Theory of Functions, Oxford (1939). 
[82] P. van der Laan, Operads and the Hopf algebras of renormalization, preprint (2003), http://www.arxiv.org/abs/math-ph/0311013.

[83] W. van Suijlekom, Multiplicative renormalization and Hopf algebras, preprint (2007), arXiv:0707.0555

[84] E. B. Vinberg, The theory of convex homogeneous cones, Trans. Amer. Math. Soc. 12 (1963), 340-403.

[85] D. Voiculescu, Dual algebraic structures on operator algebras related to free products, J. Operator Theory $\mathbf{1 7}$ (1987), 85-98.

[86] W. C. Waterhouse, Introduction to affine group schemes, Graduate Texts in Mathematics, Springer (1979).

[87] H. Zassenhaus, Über Liesche Ringe mit Primzahlcharakteristik, Abhandlungen aus dem Mathematischen Seminar der Universität Hamburg 13 No. 1 (1939), 1-100.

[88] J. Zhang, H-algebras, Adv. in Math. 89 (1991), 144-191.

[89] G. W. Zinbiel, Encyclopedia of types of algebras 2010, Proc. Int. Conf., in Nankai Series in Pure, Applied Mathematics and Theoretical Physics 9 (World Scientific, Singapore, 2012), 217-298. 